\title{
Clinical features and multidisciplinary approaches to dementia care
}

\author{
This article was published in the following Dove Press journal: \\ Journal of Multidisciplinary Healthcare \\ 14 May 2011 \\ Number of times this article has been viewed
}

\author{
Jacob HG Grand' \\ Sienna Caspar ${ }^{2}$ \\ Stuart WS MacDonald' \\ 'Department of Psychology, University \\ of Victoria, Victoria, BC, Canada; \\ ${ }^{2}$ Interdisciplinary Graduate Studies, \\ University of British Columbia, \\ Vancouver, BC, Canada
}

\begin{abstract}
Dementia is a clinical syndrome of widespread progressive deterioration of cognitive abilities and normal daily functioning. These cognitive and behavioral impairments pose considerable challenges to individuals with dementia, along with their family members and caregivers. Four primary dementia classifications have been defined according to clinical and research criteria: 1) Alzheimer's disease; 2) vascular dementias; 3) frontotemporal dementias; and 4) dementia with Lewy bodies/Parkinson's disease dementia. The cumulative efforts of multidisciplinary healthcare teams have advanced our understanding of dementia beyond basic descriptions, towards a more complete elucidation of risk factors, clinical symptoms, and neuropathological correlates. The characterization of disease subtypes has facilitated targeted management strategies, advanced treatments, and symptomatic care for individuals affected by dementia. This review briefly summarizes the current state of knowledge and directions of dementia research and clinical practice. We provide a description of the risk factors, clinical presentation, and differential diagnosis of dementia. A summary of multidisciplinary team approaches to dementia care is outlined, including management strategies for the treatment of cognitive impairments, functional deficits, and behavioral and psychological symptoms of dementia. The needs of individuals with dementia are extensive, often requiring care beyond traditional bounds of medical practice, including pharmacologic and non-pharmacologic management interventions. Finally, advanced research on the early prodromal phase of dementia is reviewed, with a focus on change-point models, trajectories of cognitive change, and threshold models of pathological burden. Future research goals are outlined, with a call to action for social policy initiatives that promote preventive lifestyle behaviors, and healthcare programs that will support the growing number of individuals affected by dementia.
\end{abstract}

Keywords: dementia, Alzheimer's disease, clinical features, multidisciplinary care, BPSD, prodromal dementia

\section{Introduction}

The clinical syndrome of dementia as a progressive deterioration of cognitive abilities and functional impairments has been recognized for over a century; however it has only been understood within the context of its current characterization in the past few decades. In the early 20th century, a shift in the conceptualization of mental illness away from causation towards disease etiology occurred, with dementia being conceived as a direct effect of age-related processes. Alzheimer's disease (AD) as a progressive neurodegenerative disorder did not emerge as a distinct entity until the mid-20th century, which led to the recognition of multiple disease states with unique etiologies. Contemporary dementia research has progressed rapidly on multiple fronts, including epidemiology, pathology, diagnosis, and treatment. As a result, four 
primary dementia groupings have been defined according to clinical and research criteria: 1) Alzheimer's disease (AD; including mixed-AD); 2) vascular dementias (VaD; including large and small vessel disease); 3) frontotemporal dementias (FTD; including Pick's disease, progressive nonfluent aphasia, and semantic dementia); and 4) dementia with Lewy bodies (DLB; including Parkinson's disease dementia [PDD]). The cumulative efforts of multidisciplinary research teams across the world have advanced our understanding of dementia beyond basic descriptions of clinical symptoms and neuropathological correlates, towards a more complete elucidation of risk factors and underlying pathobiological disease mechanisms and, importantly, have led to targeted treatments, management, and care.

The needs of individuals affected by dementia are extensive, and the management of the clinical syndrome is complex, extending beyond traditional bounds of medical practice. Whereas medicine has historically focused on the effects of disease on the patient, dementia care requires a broader focus extending to family members, caregivers, and support networks. This broad-based approach to the care and management of dementia involves consideration of the quality of life for affected individuals to a greater extent than many other disease states. Dementia is characterized by significant impairments in multiple cognitive domains, functioning, and behavior, and places a tremendous burden not only on individuals, but also on society. Early detection and management may prevent overuse of costly healthcare resources and allow affected individuals and caregivers time to prepare for future medical, financial, and emotional challenges. Many of the most effective management approaches are nonpharmacologic, and provided by healthcare professionals from multiple disciplines. We are now entering an era of dementia care that will be based upon the identification of early disease markers and potentially modifiable risk factors, along with the application of novel diagnostic tools, treatment modalities, and care practices.

In this review, we briefly summarize the current state of knowledge and directions of research for dementia epidemiology, its clinical presentation, multidisciplinary approaches to care, treatment options, management challenges, and future research goals.

\section{Population aging and dementia epidemiology}

Population aging and increased life expectancy has become a worldwide epidemiological phenomenon. Global projections for the number of older individuals ( $\geq 65$ years) are expected to increase from 420 million in 2000 , to nearly 1 billion by 2030 , with a corresponding increase in the proportion of older individuals rising from $7 \%$ to $12 \% .{ }^{1,2}$ Developing countries will see the greatest rise in absolute numbers of older individuals, and will contribute to worldwide population aging with an increase from $59 \%$ to $71 \%{ }^{3}$ Dementia is strongly associated with increasing age, and consequently neurodegenerative disorders are anticipated to pose significant challenges to public healthcare systems worldwide in the coming decades.

The prevalence of dementia is also rising, particularly $\mathrm{AD}$, which is the most common form of dementia accounting for approximately $60 \%$ of all cases. ${ }^{4,5}$ Worldwide, the global prevalence of dementia is estimated to be $3.9 \%$ in individuals over the age of 60 years, with regional prevalence rates of $1.6 \%$ in Africa, $4.0 \%$ in China and Western Pacific Regions, 4.6\% in Latin America, 5.4\% in Western Europe, and 6.4\% in North America. ${ }^{3}$ Greater than 25 million people in the world are currently affected by dementia syndromes, with most individuals suffering from AD and vascular dementia (VaD) (Figure 1). ${ }^{4-6}$ Approximately 5 million new cases are estimated to occur each year, and the number of individuals with dementia is expected to double every 20 years (Figure 2). 2,3,6

\section{Risk factors, clinical presentation, and diagnosis of dementia}

The clinical syndrome of dementia generally follows three primary expressions. ${ }^{7-10}$ First, a neuropsychological component consisting of a range of cognitive impairments, often including: memory impairments (declarative and procedural memory problems), aphasias (either receptive or expressive language difficulties), apraxias (inability to carry out directed coordination of movements despite intact sensory and motor nervous systems), agnosias (inability to recognize specific elements of an individual's environment or self), attentional difficulties (including sustained and divided attention), and executive functioning impairments (including difficulties

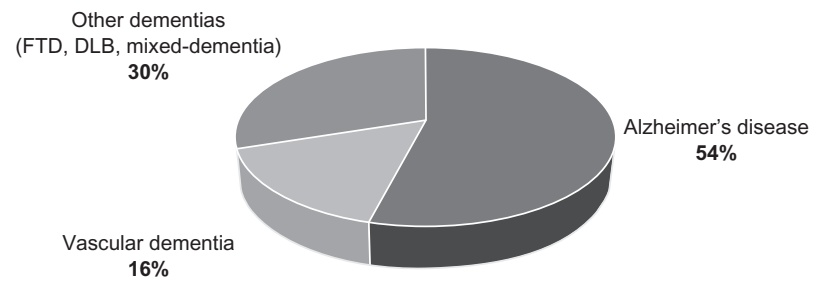

Figure I Worldwide subtypes of late onset dementia ( $\geq 65$ years). Copyright (C) 2007, Elsevier Inc. Adapted with permission from Brookmeyer et al. ${ }^{6}$ Abbreviations: DLB, dementia with Lewy bodies; FTD, frontotemporal dementias. 

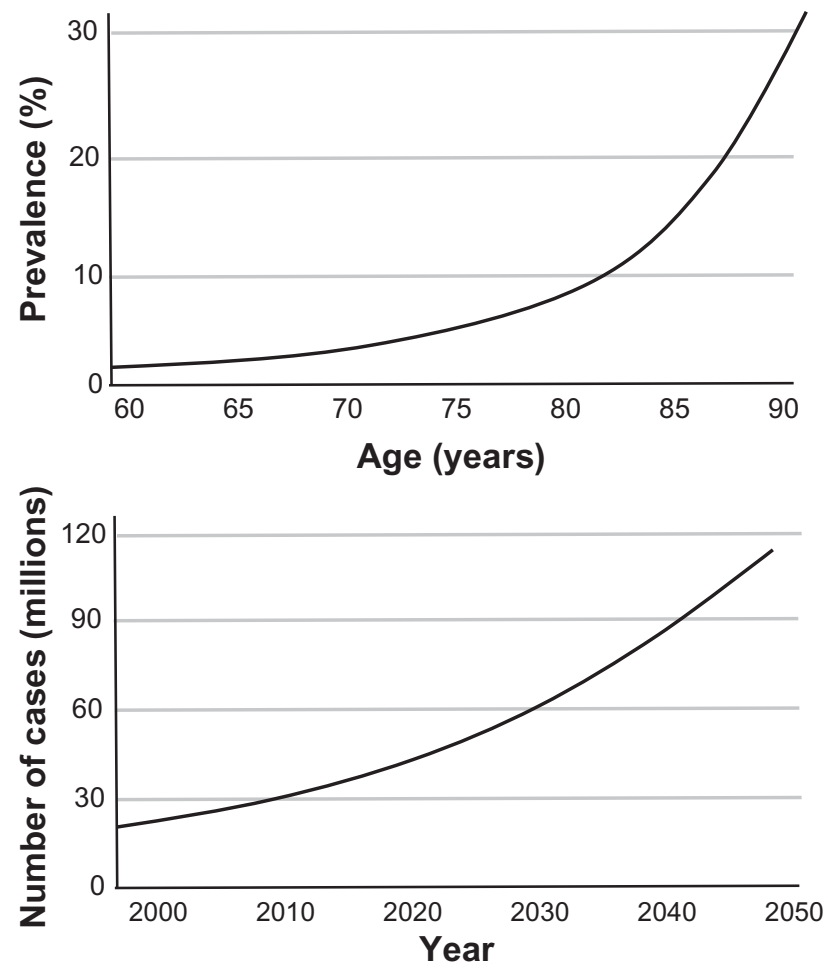

Figure 2 Global prevalence of Alzheimer's disease as a function of age and total number of worldwide cases per year.

Copyright @ 2006, Lancet Publishing Group. Adapted with permission from Ferri et al. ${ }^{3}$

with abstraction, cognitive flexibility, inhibition, planning, organizing, and adaptation to novel stimuli). The second primary expression of this syndrome is a neuropsychiatric element with associated symptoms and behavioral disturbances. These psychiatric features are present in a substantial proportion of affected individuals, and are commonly referred to as behavioral and psychological symptoms of dementia (BPSD). Common BPSD disturbances include depression, paranoid ideation, delusions, hallucinations, aggression, and wandering. ${ }^{11}$ The third primary clinical expression includes deficits in activities of daily living (ADL). ${ }^{8}$ In the early stages of dementia, impaired instrumental activities of daily living (IADL) may be manifest including self-neglect of diet, personal hygiene, and common household tasks. Towards the later stages of dementia, basic activities of daily living (BADL) are often impaired, presenting with obvious problems in dressing, eating, and bathing. ${ }^{12,13}$

This triad of features is common to most dementias, with the differentiation of subtypes based on clinical presentation, the presence of comorbid symptoms, and other aspects of the individual's history and examination. ${ }^{7,8,14}$ Individuals with dementia tend to present to specialist healthcare services (eg, neurologist, neuropsychologist, memory disorders clinic) only when symptoms begin to interfere with everyday activities and functioning. Depending upon the stage of disease progression, the patient is often unable to provide an accurate history and may deny any presence of impairments. Therefore, a detailed history from a reliable primary informant (eg, family, caregiver) is essential to provide collateral information of previous baseline functioning, symptom onset, and supportive evidence of changes in cognitive and behavioral functioning. ${ }^{15}$ Depending on the dementia subtype, there is wide variability in rates of decline from person to person and in the rapidity with which the disease process develops. Nevertheless, all dementias are degenerative and progressive. Early detection and the differential diagnosis of dementia subtype, disease complexity, and sequelae requires skilled clinical judgment and is based on multiple sources of information (see Figure 3). ${ }^{16}$

\section{Mild cognitive impairment}

Clinical AD is often preceded by a prodromal phase commonly referred to as mild cognitive impairment (MCI) ${ }^{17}$ Despite several operational definitions, a diagnosis of MCI is generally applied to individuals with a primary complaint and objective demonstration of cognitive impairments in one or more domains (usually memory difficulties), but with relatively preserved ADL. ${ }^{9,17}$ Debate continues regarding the most useful way to operationalize the clinical and research criteria for a diagnosis of MCI. ${ }^{18-24}$ Most MCI classifications require a subjective memory complaint, informant corroboration of memory difficulties, relatively intact ADL, and normal or mild problems in nonmemory cognitive functioning. ${ }^{9}$ More stringent classifications specify unique cutoff points for the severity of memory impairment on standardized neuropsychological tests (eg, performance $<1.5 \mathrm{SD}$ below the mean). ${ }^{25}$ The clinical utility of MCI as a diagnostic classification is evidenced by estimates of increased risk for conversion to AD. ${ }^{24,26,27}$ While there are considerable individual differences in trajectories of change, in general, individuals with MCI progress to $\mathrm{AD}$ at a rate of $10 \%$ to $25 \%$ per year, compared with healthy elderly controls who progress at a rate of approximately $1 \%$ to $2 \%$ per year. ${ }^{9,18,24,28}$

Individuals with $\mathrm{MCI}$ tend to demonstrate impaired performance on neuropsychological tests of memory. ${ }^{9}$ This is in part a reflection of diagnostic criteria such that individuals with mild impairment in other cognitive domains may be missed. The pattern of memory decline observed in MCI appears to reflect a degradation of information in memory storage and retrieval, rather than an impairment of information encoding. ${ }^{25}$ The storage deficit is demonstrated in the loss of information over time, with minimal or no improvement in performance when assessed using tests 


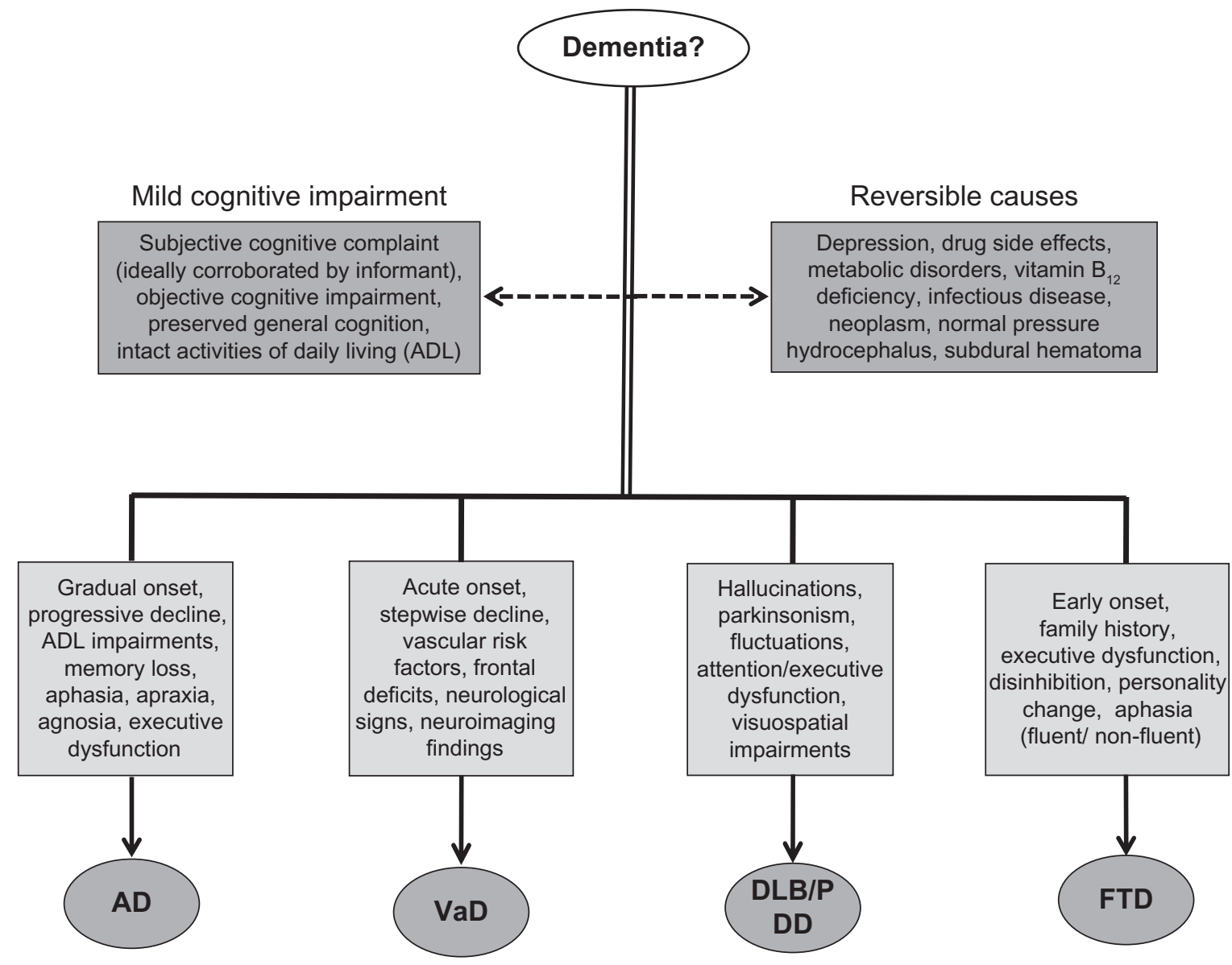

Figure 3 Differential diagnostic considerations for dementia.

Abbreviations: AD, Alzheimer's disease; DLB, dementia with Lewy bodies; FTD, frontotemporal dementia; MCI, mild cognitive impairment; PDD, Parkinson's disease dementia; $\mathrm{VaD}$, vascular dementia.

of recognition. This pattern of decline is generally consistent with that observed for individuals with $\mathrm{AD}$, and is thought to be evidence that $\mathrm{MCI}$ is a preclinical stage of $\mathrm{AD}$.

Recent research has revealed early changes associated with MCI including difficulties with executive functioning and increased apathy., ${ }^{9-31}$ Individuals with MCI have also been found to have difficulties dealing with financial matters, including problems related to cash management and bill payment. ${ }^{32}$ Given the overall high level of functioning, individuals with these difficulties tend to remain undetected, thereby making them more vulnerable for potential future decline. ${ }^{29,31}$ Therefore, sensitive measures of memory, executive functioning, and personality shifts may help to improve prediction of conversion from MCI to more severe forms of dementia. ${ }^{9,29,31}$

\section{Alzheimer's disease Risk factors}

$\mathrm{AD}$ is the most common form of dementia, accounting for an estimated two-thirds of dementia cases in individuals aged 65 years or older. ${ }^{4,5}$ Age is the most consistent risk factor for $\mathrm{AD}$, presumably due to lifelong exposure to various forms of neural damage, including minor vascular events, white matter disease, and inflammation. ${ }^{5,7,10,18}$ Other potential risk factors include: level of education (higher education potentially conferring increased brain and cognitive reserve), gender (increased risk for female sex), family history of dementia (particularly for first degree relatives), genetic factors (familial forms of $\mathrm{AD}$ are associated with mutations in three autosomal dominant genes: presenilin $1(P S-1)$, presenilin $2(P S-2)$, and amyloid precursor protein $(A P P)$, which are thought to work through increased production of $A \beta$ peptides; and polymorphisms of apolipoprotein E, particularly Apoe4 which is associated with 2 to 3 times (heterozygotes) and 6 to 8 times (homozygotes) increased risk of developing $\mathrm{AD}$ ), vascular risk factors (including hypertension, hypercholesterolemia, diabetes mellitus), mood disorders (particularly depression), and psychosocial lifestyle factors 
(including reduced intellectual, physical, recreational, and social engagement). ${ }^{7,33}$

\section{Clinical features}

AD presents with an insidious onset and progresses gradually and inexorably. ${ }^{34}$ Therefore, identifying specific time points corresponding to initial symptom onset is often difficult. ${ }^{28}$ The diverse clinical features of cognitive impairment in AD reflect dysfunction of multiple regions of the cerebral cortex. Memory decline is commonly the presenting complaint in $\mathrm{AD}$, and represents a cardinal feature of the disease. The memory changes associated with $\mathrm{AD}$ are characterized as storage deficits, with rapid forgetting and poor delayed memory recall. In mild-AD, core episodic memory impairments are observed, reflecting early medial temporal lobe and limbic involvement. ${ }^{8,35}$ For example, AD individuals may have difficulty recalling details of recent events, personal conversations, or specific elements of a task they may be performing (eg, preparing a meal). Often, they will ask the same question multiple times and may deny their repeated line of questioning. ${ }^{8}$ Individuals with $\mathrm{AD}$ also tend to confabulate and generate inaccurate responses to questions. ${ }^{36}$ On neuropsychological tests of word recall, AD patients produce more intrusions of incorrect word responses and inaccurately recognize words as being part of the original list (false positive responses) compared with individuals with other subtypes of dementia. ${ }^{9}$ In general, deficits in declarative memory (episodic memory) are prominent at the disease outset, with more severe memory impairments (semantic memory) emerging later in the disease course..$^{35,37}$

Orientation to time and place are also disrupted early in the disease, probably because these abilities require effective learning and encoding of information from recent memory. As the disease progresses, orientation worsens to include problems identifying familiar places, family members, or other people well known to the individual. ${ }^{9}$

Procedural memory may also decline as the disease worsens, resulting in reduced ADL. Problems may include dressing or using common utensils and can be characterized as an ideomotor apraxia. On testing, these maladaptive behaviors are commonly seen when AD individuals use their finger as a toothbrush rather than pantomiming holding the toothbrush as requested. ${ }^{14}$

Language impairment, although generally preserved early in the disease, is also a prominent clinical feature. Initial signs of language dysfunction often include reduced expressive language (conversational output), word-finding difficulties, and limited vocabulary. Category fluency (eg, naming animals or furniture) tends to be more impaired than semantic fluency (eg, naming words that begin with a particular letter), perhaps because category fluency is dependent on access to temporallobe semantic stores. ${ }^{38,39}$ As the disease progresses, language difficulties become more pervasive and nonfluent. Spoken language may become less meaningful with an increase in comprehension difficulties, eventually resulting in global aphasia late in the course of illness. ${ }^{14}$

Although memory decline and language impairment are prominent findings in $\mathrm{AD}$, involvement of other cognitive domains is also common. Visuospatial dysfunction may manifest as impaired driving ability, getting lost, and difficulty producing drawings of figures. Problems with mathematical calculations may impair the individual's ability to use money and balance their finances. Executive dysfunction results in deficits in problem solving, abstraction, reasoning, decision making, and judgment, particularly for novel situations. For example, AD individuals often display difficulty executing complex tasks, such as planning a vacation or negotiating a business deal. ${ }^{8}$

In addition to cognitive impairments, behavioral and psychiatric symptoms are common in AD. Depression rates vary by severity and disease stage, and can occur in up to $50 \%$ of AD individuals. ${ }^{40}$ Early in the disease course, depression may be attributed to awareness of the individual's cognitive changes. In the later stages, features related to apathy may present, but are often confused with depression, particularly by caregivers. Apathy is associated with significant caregiver distress and greater use of healthcare services in AD. ${ }^{41}$ Psychosis generally occurs later in the disease course. Delusions are predominantly paranoid in nature, with fears of personal harm or mistreatment, theft of personal property (usually related to financial matters), and marital infidelity. Hallucinations are less common than delusions, and tend to be visual. Other behavioral symptoms include agitation, wandering, and sleep disturbances. The primary treatable behavioral and psychiatric symptoms in AD include psychosis, agitation, depression, anxiety, and insomnia. ${ }^{42}$

\section{Diagnosis}

The diagnosis of AD is based on identification and evaluation of features of the individual's history and clinical exam that are suggestive of the disease, while excluding other causes of dementia through the use of laboratory tests and neuroimaging. The two most commonly used diagnostic criteria for AD are those of the Diagnostic and Statistical Manual of Mental Disorders, Fourth Edition Revised (DSM-IV-TR), ${ }^{43}$ and those created by the National 
Institute of Neurological and Communicative Disorders and Stroke and the Alzheimer's Disease and Related Disorders Association (NINCDS-ADRDA) joint task force (Table 1). ${ }^{44}$ The DSM-IV-TR criteria require an insidious onset with progressive decline of cognitive function that results in impairment of social and occupational functioning, along with memory impairment and at least one other cognitive deficit that cannot be attributed to other psychiatric, neurological, or systemic disease. The NINCDS-ADRDA criteria classify AD according to three levels of diagnostic certainty: definite, probable, and possible. A definite diagnosis of AD is restricted to individuals who meet the clinical criteria for probable AD and have corroborating neuropathological evidence of the disease (by biopsy or autopsy). Probable AD is the most definitive level of diagnostic certainty, without pathological confirmation. Probable AD requires a progressive decline of memory, along with at least one other cognitive domain that is confirmed through clinical examination and neuropsychological testing. The individual must have a preserved level of consciousness, with exclusion of other conditions that may account for the symptoms. Additional features supportive of a probable AD diagnosis include: progressive decline of language functions, praxis, visual recognition, and impaired ADL, with normal results on laboratory tests (eg, cerebrospinal fluid examination), electroencephalogram, and neuroimaging. A diagnosis of possible $\mathrm{AD}$ is appropriate when the clinical course of cognitive decline is atypical, when focal neurological findings are present, or when comorbid disorders exist that may account for the dementia-like symptoms. The NINCDS-ADRDA criteria are widely used for research and clinical diagnosis, and have been shown to have a predictive accuracy of up to $90 \%$. $^{35,45,46}$

\section{Vascular dementia}

\section{Risk factors}

$\mathrm{VaD}$ is a clinical syndrome of acquired cognitive and functional impairments resulting from the effects of cerebrovascular disease. Due to the multiple etiologies of cerebrovascular disease, $\mathrm{VaD}$ can be characterized across a range of neurological and neuropsychological features that vary in presentation by onset (insidious or abrupt) or course (progressive, remitting, or static) of the dementia syndrome. ${ }^{47}$ Alternate definitions of $\mathrm{VaD}$ and a lack of universally accepted diagnostic criteria make it difficult to accurately estimate global prevalence rates. Nevertheless, general trends have been observed with prevalence estimates of $1 \%$ to $4 \%$ in individuals over 65 years, and worldwide prevalence rates of $15 \%$ to $22 \%{ }^{5,48}$ The association of stroke with cognitive impairment and vascular dementia has been demonstrated in a number of epidemiological, clinical, and neuropathologic studies. ${ }^{49-51}$ Comorbid disorders leading to hypoxemia (seizures, cardiac arrhythmia) have been shown to be potential risk factors for dementia in stroke patients. ${ }^{51}$ Other clinical factors associated with a higher risk of $\mathrm{VaD}$ include increasing age, lower education, cortical atrophy, previous vascular events, and atherosclerosis. ${ }^{50,51}$ The association between hypertension and cognitive impairment remains a widely investigated area of research. Most longitudinal studies have found a significant association between elevated blood pressure and the occurrence of dementia 10 to 15 years later. In this association, diastolic pressure appears to be of greater importance than systolic pressure. ${ }^{52} \mathrm{~A}$ history of coronary artery disease, myocardial infarction, diabetes mellitus, smoking, and hyperlipidemia are additional risk factors for $\mathrm{VaD} .{ }^{51-53}$ The underlying mechanism by which cerebrovascular risk factors lead to cognitive impairment

Table I Summary of DSM-IV and National Institute of Neurological and Communicative Disorders and Stroke and the Alzheimer's Disease and Related Disorders Association (NINCDS-ADRDA) criteria for the diagnosis of Alzheimer's disease (AD)

\begin{tabular}{|c|c|}
\hline DSM-IV criteria & NINCDS-ADRDA criteria \\
\hline - Insidious onset with progressive decline of cognitive & - Probable AD \\
\hline function resulting in impairment of social or occupational & - Deficits in two or more domains of cognition \\
\hline functioning from a previously higher level & - Progressive decline of memory and other cognitive functions \\
\hline - Impairment of recent memory and at least one of the & - Preserved consciousness \\
\hline following cognitive domains: & - Onset between ages 40 and 90 years \\
\hline - Aphasia & - Absence of systemic or other brain disease that could account \\
\hline - Apraxia & for symptoms \\
\hline- Agnosia & - Possible AD \\
\hline - Executive functioning (planning, organizing, sequencing, & - Atypical onset, presentation, or clinical course of dementia \\
\hline abstraction) & - Presence of another illness capable of producing dementia \\
\hline - Cognitive deficits are not due to other neurological, psychiatric, & - Definite AD \\
\hline toxic, metabolic, or systemic diseases & - Clinical criteria for probable AD \\
\hline - Cognitive deficits do not occur in the setting of a delirium & - Tissue diagnosis by autopsy or biopsy \\
\hline
\end{tabular}


and dementia remains to be fully elucidated. Most studies suggest that cerebrovascular risk factors are associated with an increased prevalence of cerebral white matter abnormalities, whole brain atrophy, and silent stroke. ${ }^{54}$ Associations have also been found between the extent of brain atrophy or white matter hyperintensity volumes and decreased cognitive abilities. Therefore, growing evidence suggests that cerebrovascular disease, even in the absence of clinical symptoms, may lead to subsequent cognitive impairment. ${ }^{55-57}$

\section{Clinical features}

Vascular dementia is a diagnostic classification used to describe a constellation of clinical features, including cognitive and functional impairments. ${ }^{58}$ Three vascular syndromes are commonly cited in the literature: multi-infarct dementia, strategic single infarct dementia, and small vessel disease with dementia.

Multi-infarct dementia (also known as post-stroke dementia) is the most common form of vascular dementia. ${ }^{59}$ It is attributable to multiple infarcts with occlusion, usually in large vessels, that cause cortical and subcortical infarcts. Occlusion of these arteries occurs as a result of atherosclerotic thrombosis or cardiac embolization, and typically has an abrupt onset with stepwise progression. Multi-infarct dementia is characterized by variable impairment across several cognitive domains. ${ }^{60}$ Individuals may present with severe deficits in certain domains, while other cognitive abilities may be spared and remain relatively intact. With recurrent stroke episodes, greater areas of cognitive functioning become impaired and the dementia syndrome emerges. $\mathrm{VaD}$ individuals often present with lateralized sensorimotor changes, including hemiparesis, hemisensory loss, visual field disturbances, and pathological reflex asymmetries. Agnosia and apraxia may also be part of the clinical features in multi-infarct dementia. ${ }^{60}$

In strategic single infarct dementia, ischemic damage is focal and involves functionally important cortical and subcortial regions including the thalamus, basal ganglia, angular gyrus, and frontal white matter. ${ }^{54}$ For example, an infarct in the angular gyrus may result in clinical features such as fluent aphasia, alexia with agraphia, and spatial disorientation. Single lesions in the carotid, anterior, middle, and posterior cerebral arteries are known to give rise to clinical dementia symptoms with a number of neurological and neurobehavioral features. ${ }^{51}$ Clinical evidence of stroke may or may not be present, but the onset of dementia is generally abrupt with a stepwise decline in cognitive functioning.
Small vessel disease with dementia refers to a syndrome in which occlusion of small vessels causes lesions of subcortical structures, including the thalamus, basal ganglia, internal capsule, and sub-hemispheric white matter. Disruption of these structures results in a form of subcortical dementia. This state is characterized by insidious onset of symptoms including psychomotor slowing, memory impairments, changes in speed, and neuropsychiatric features such as depression and apathy. Neurological features include parkinsonism, ataxia, and urinary incontinence. ${ }^{61}$ Individuals with small vessel disease with dementia often present with slow, progressive, dementia-like features, often without clinical symptoms of stroke. Cerebral autosomal dominant arteriopathy with subcortical infarcts and leukoencephalopathy (CADASIL) is thought to be the hallmark disease associated with this clinical syndrome. ${ }^{62}$

\section{Diagnosis}

Several clinical criteria currently exist for the diagnosis of $\mathrm{VaD}$ dementia. The Hachinski Ischemic Scale (HIS) is a commonly used set of criteria for $\mathrm{VaD}$ that is easily applied in clinical practice. ${ }^{63}$ The HIS is based on 13 features used to obtain a total ischemic score. The current modified HIS emphasizes a subset of clinical symptoms as a means of distinguishing VaD from AD. ${ }^{64}$ The general features of the scale include: abrupt onset, prior history of stroke, focal neurological signs, focal neurological symptoms, stepwise deterioration, somatic complaints, emotional ability, and history of hypertension. Despite a sensitivity and specificity of $70 \%$ to $80 \%$ in separating VaD from $\mathrm{AD}$, the HIS is limited in its clinical use by not integrating neuroimaging information into the diagnostic process. The DSM-IV-TR approach utilizes clinical evidence of stroke-based signs from the neurological examination or neuroimaging evidence for infarcts as the primary basis for a diagnosis of $\mathrm{VaD}^{43}$ These criteria do not require that the dementia be temporally associated with the occurrence of stroke, which in effect decreases its specificity and clinical utility. Another definition of $\mathrm{VaD}$ is based on the World Health Organization's International Statistical Classification of Diseases, 10th Revision (ICD-10), which requires a temporal link between a stroke event and dementia, along with signs of stroke on neurological examination or imaging evidence of an infarct. ${ }^{65}$ A final diagnostic classification for $\mathrm{VaD}$ is based on the National Institute of Neurological Disorders and Stroke and Association Internationale pour la Neurosciences (NINDS-AIREN). These criteria are the most widely used for $\mathrm{VaD}$ in clinical trials research and 
emphasize the heterogeneity of vascular syndromes and white matter lesions. The NINDS-AIREN criteria require neuroimaging evidence of lesions in key areas involved in $\mathrm{VaD}$ (frontal, temporal, or parietal cortex, thalamus, basal ganglia), along with critical abnormal white matter signals $\left(>25 \%\right.$ of total white matter volume) ${ }^{47}$

\section{Frontotemporal dementia}

The clinical and pathological understanding of frontotemporal dementia (FTD) has undergone considerable changes in the past decade. Frontotemporal lobar degeneration (FTLD) is a descriptive term that encompasses a variety of clinical syndromes associated with nonAlzheimer pathology and arises from degeneration of the anterior hemispheres. ${ }^{66}$ This heterogeneous group of clinical syndromes includes frontotemporal dementia (FTD), FTD with motor neuron disease (FTD/MND), progressive nonfluent aphasia (PNFA), semantic dementia (SD), and progressive apraxia. ${ }^{66}$ The most commonly occurring syndrome is frontotemporal dementia (FTD) which is characterized by profound alterations in personality and behavior, and is associated with bilateral atrophy of the frontal and anterior temporal lobes. ${ }^{67}$ Panels of expert international working groups have developed a number of clinical diagnostic criteria for FTD. ${ }^{67-69}$ The characteristic feature of FTD is a slowly progressive dementia that presents with either predominant behavioral or language disturbances (Table 2). ${ }^{70}$ FTD tends to have a younger age of onset than other dementias, typically between

Table 2 Common clinical presentations of frontotemporal dementia

\begin{tabular}{ll}
\hline Presentation & Clinical features \\
\hline Behavioral & - Impulsive behaviors and disinhibition \\
& (eg, inappropriate sexual behaviors) \\
- & Change in personality (eg, indifference, apathy, \\
& mental inflexibility) \\
- Poor judgment and poor insight into consequences & of behaviors (eg, financial risk taking) \\
- Repetitive, compulsive, stereotypic behaviors & (eg, hyperorality) \\
- Loss of personal hygiene (eg, wearing same clothes & for multiple consecutive days \\
- Loss of social graces/insight (eg, making jokes during & a funeral) \\
- Non-fluent aphasia $\rightarrow$ with anomia, agrammatism, \\
phoemic paraphrasias, impaired word repetition, \\
- alexia, agraphia \\
- Fluent-aphasia $\rightarrow$ with early loss of word meaning \\
(ie, empty speech), poor comprehension, semantic \\
paraphrasias, prosopagnosia, associative agnosia
\end{tabular}

Copyright (C) 2010, Lippincott Williams \& Wilkins. Modified with permission from Arvanitakis. ${ }^{70}$
45 to 65 years, and approximately $20 \%$ to $30 \%$ of cases are familial and may be associated with mutations in the progranulin or MAPT gene. ${ }^{71}$ Prevalence studies of FTD are variable, with estimates ranging between 3.6 to 15.0 per 100,000 individuals. $^{72}$

Of the two FTD clinical presentations, the more common form is that of a behavioral disturbance, which is associated with profound changes in personality. Typical behavioral changes include apathy, lack of initiation, disinhibition (verbal, physical, and sexual disinhibition), perseveration, tactlessness, lack of social graces, emotional blunting, and loss of insight. FTD individuals tend to be unaware of or unconcerned about the social, occupational, and financial consequences of their behavioral changes. ${ }^{73}$ Cognitive deficits tend to occur in the domains of attention, abstraction, shifting, planning and problem solving, reflecting an executive dysfunction. $^{9}$

The less common presentation of FTD is that of a progressive and relatively isolated language disturbance. This language presentation can either be that of a difficulty with expression and naming, suggestive of a primary progressive aphasia (usually a nonfluent aphasia), or that of difficulty with word meaning, consistent with a semantic dementia. ${ }^{68}$ Progressive nonfluent aphasia presents with a gradual disturbance of expressive language with effortful speech production, phonological and grammatical errors, and word retrieval difficulties, ultimately followed by later mutism. Reading and writing can also be impaired. Other cognitive functions are usually spared and these individuals have relatively preserved social skills early in the disease. ${ }^{9}$ Semantic dementia presents with progressive impairment of comprehension of words and naming in the context of fluent, effortless and grammatically correct speech output. Memory functions are relatively preserved, along with word repetition, reading skills, and mathematical abilities. ${ }^{9}$

The different clinical presentations of FTD are thought to be associated with the initial focal pattern of cortical atrophy of the frontal lobes. However, as the disease progresses a more global pattern of cognitive impairment emerges. ${ }^{68} \mathrm{On}$ examination, early signs include the presence of a grasp, snout, or palmomental reflex. Further, disinhibition and perseverative behaviors are often observed. The presence of additional neurologic abnormalities may suggest early motor neuron disease (MND) or parkinsonism. There are no widely accepted bedside cognitive or behavioral tests for FTD, and measures of global cognition like the Mini-Mental State Examination (MMSE) are insensitive to change in behavior, 
aphasia detection, and evaluation of executive function. If FTD is suspected, a more extensive neuropsychological battery should be administered and the individual should be monitored closely.

\section{Dementia with Lewy bodies}

Dementia with Lewy bodies (DLB) usually presents in older adulthood between the ages of 60 and 90 years. The epidemiology of DLB remains to be fully elucidated along with potential risk factors for the disease, but to date no significant gender or ethnic differences in prevalence have been identified. ${ }^{74}$ DLB is a neurodegenerative disorder, thus progressive and functionally disabling cognitive impairment is a central feature. Individuals with DLB exhibit a combination of cortical and subcortical impairments with significant attentional deficits and executive and visuospatial dysfunction. ${ }^{9}$ Compared to $\mathrm{AD}$, there may be relative sparing of memory functions especially in the early stages of DLB. ${ }^{75}$ A recent international consortium has resulted in revised criteria for the clinical diagnosis of DLB, incorporating new information about the core clinical features of the disease. These core features include fluctuating cognition, recurrent visual hallucinations, and spontaneous parkinsonism. ${ }^{76}$

Fluctuations in cognitive function with pronounced variations in attention and alertness can be difficult to evaluate and operationalize, but corroborative reports from primary caregivers can help to clarify these essential clinical features (eg, daytime drowsiness, prolonged staring spells, periods of marked confusion, disorganized speech). ${ }^{76}$ Cognitive fluctuations are reported across varying time periods, but generally take place for a minimum of 30 minutes and can last as long as several days. ${ }^{9}$

Visual hallucinations in DLB tend to be recurrent, vivid, and well-formed. The hallucinations tend to emerge early in the disease course, and are therefore a useful diagnostic feature. The content of the hallucinations usually consist of humans or animals, but can be more abstract in nature. Visual illusions and delusions, which typically have a paranoid quality, are also commonly observed. Auditory, tactile, or olfactory hallucinations are rare. Functional neuroimaging studies have demonstrated altered patterns of activation in the visual cortex, along with increased number of Lewy bodies in the anterior and medial temporal lobe of individuals with DLB who report hallucinations. ${ }^{77}$ Other common neuropsychiatric features include depression, apathy, and anxiety. Agitation and aggressive behavior tend to occur late in the course of the disease.
Extrapyramidal signs, including bradykinesia, facial masking, and rigidity, are the most frequent signs of parkinsonism in DLB. Resting tremor is distinctly uncommon, and parkinsonism is usually bilateral and occurs with the onset of dementia. There is often more axial rigidity and facial masking in DLB than is typically seen in idiopathic Parkinson's disease. ${ }^{76}$

An important suggestive clinical feature of DLB includes rapid eye movement (REM) sleep behavioral disturbances. Affected individuals often act out their dreams by screaming and kicking, which can cause injuries to themselves and those around them. The dreams tend to have a chasing or attacking theme, and their content usually matches the exhibited behavior. REM sleep behavior disturbances often begin years before the onset of other cognitive or motor symptoms. ${ }^{78}$

Other suggestive features of DLB include severe neuroleptic sensitivity, and low dopamine transporter uptake in the basal ganglia on functional neuroimaging. Additional supportive features that commonly occur in DLB include repeated falls and syncope, transient, unexplained loss of consciousness, severe autonomic dysfunction, hallucinations in other modalities, systematized delusions, relative preservation of medial temporal lobe structures on structural neuroimaging, reduced occipital activity on functional neuroimaging, prominent slow wave activity on electroencephalogram, and low uptake myocardial scintigraphy. ${ }^{76}$

\section{Multidisciplinary team approach to dementia care}

An integrated multidisciplinary approach to diagnosing and managing dementia is highly recommended in clinical practice because no single healthcare specialty has the expertise to deal with the complex range of cognitive, physical, social, and emotional problems associated with dementia. Managing dementia presents unique challenges to the practicing clinician, and effective care hinges on a collaborative team approach. This approach relies less on standard pharmacologically based medical practice, and more on the integration of therapies from a wide range of healthcare providers and community professionals. To implement this approach effectively, the clinician must understand the disease processes involved and the clinical presentation of dementia, and also appreciate the caregiving experience and how it affects patient care. The clinician must be informed about the resources available to meet the medical, social, and emotional needs of the patient in the context of their family and community. Using a multidisciplinary team approach can be complex, 
but it benefits both the patient and the clinician. It allows clinicians to focus on the issues most relevant to their area of expertise, and it facilitates management of patient problems that are likely to require valuable healthcare resources. ${ }^{15}$

\section{Multidisciplinary team structure}

The best approach to the care of individuals affected by dementia includes support from multiple sources. These may be integrated, parallel, or a combination of both. It is ideal for patients and caregivers to seek a blend of multidisciplinary services that come from various healthcare providers, social service agencies, and professionals from outside the field of healthcare. Multidisciplinary teams vary in their composition and may be structured either formally or informally. Team construction is usually dictated by resources, including time, availability, finances, and geographic location. Regardless of structure, successful teams are characterized by a shared commitment to quality care and an appreciation for the contributions of each team member. ${ }^{79,80}$

Multidisciplinary teams involved in dementia care tend to be based primarily on the availability of service resources, in addition to the social and cultural context of the community. Team members often include neurologists, geriatricians, neuropsychologists, nurse practitioners, physical/occupational therapists, nutritionists, and social workers. ${ }^{15}$

The neurologist or geriatrician's primary responsibility is the diagnosis of dementia using validated criteria and practice guidelines. Following diagnosis, a prognosis is determined and a treatment plan is formulated to address the disease and underlying symptoms. ${ }^{15}$ When diagnostic uncertainty exists, referral to a subspecialist may be useful to refine the diagnosis and help develop a plan of care. Dementia subspecialists may include behavioral neurologists, geriatric psychiatrists, or neuropsychologists. The role of a neuropsychologist is to interpret quantitative cognitive performance data based on the results of psychometric testing. Many neuropsychologists also provide ongoing therapy and support to the dementia patient, and their family members. ${ }^{9}$

The nurse's primary role is to assess and manage the dementia patient and their caregivers' responses to the disease process. This includes monitoring symptom presentation, responding to medication issues, providing education and relevant information to family members, and assisting them in preparing for disease progression. Dementia frequently results in immobility, decreased physical conditioning, loss of muscle strength and tone, and poor coordination. Impaired ambulation is common and increases the risk of falls and injury. Physical therapists can assist dementia patients to optimize their physical conditioning and maintain safe mobility, helping to prolong independent living and the need for institutionalization. ${ }^{81}$

Another important factor in maintaining the dementia patient's home independence is their ability to manage ADL. Occupational therapists help the patient and their caregivers to adapt to the patient's diminished ability to deal with challenges faced in daily living. Use of devices to assist with toileting, eating, dressing, and home management can be recommended and demonstrated by an occupational therapist. As patient independence is enhanced, caregiver burden decreases ${ }^{82}$ Cognitive impairment related to dementia also places the patient at increased risk for malnutrition and dehydration. In later, more severe stages of dementia, the patient often experiences sensory-motor impairments with ensuing loss of ability to swallow or eat independently. Weight loss then becomes a significant concern as the disease progresses. Comorbid conditions common to older individuals such as diabetes and coronary heart disease also make dietary management more challenging. Dietary intake also extends beyond medical concerns, and becomes an important quality of life consideration. ${ }^{83}$

Social workers base their practice on the philosophy that the individual is part of a larger system, and they are prepared to assist the patient and their family in identifying and acquiring the resources needed to manage the burden of chronic illness. ${ }^{84}$ In nearly all cases, patients with dementia and their caregivers will require access to social services including community programming, respite care, financial services, counseling support groups, and crisis management. Therefore, it is often recommended that a referral to a social worker be initiated at the time of formal diagnosis of dementia. ${ }^{15}$ As the disease progresses, increasing levels of support will be required. The social worker is able to help anticipate future needs, facilitate the acquisition of services, and navigate the patient and their caregivers through the extended healthcare system. For individuals with dementia, the home environment has been found to promote a sense of personhood, normalcy and continuity in the face of disconnection that is often experienced as a result of multiple losses in functional, cognitive, and social domains of their lives.$^{85}$ Consequently, 'aging in place' is considered to be the preferred choice for many older adults to help maintain independence. ${ }^{86,87}$ However, individuals with dementia often require care within a structured, institutionalized environment. Caregivers' reasons for placement into institutionalized care are diverse and often include: 1) the need for more skilled care and more assistance, 2) the caregivers' health, 
3 ) the presence of behavioral and psychological symptoms of dementia, 4) caregiver burden, and 5) use of communitybased services. ${ }^{88}$ Given the practical and emotional challenges involved in transitioning from a home environment to an institutionalized care facility, the social worker can help guide the dementia patient and their family members through this difficult process.

\section{Managing and treating dementia}

Current clinical practice encourages open discussion of the diagnosis of dementia with patients and their caregivers to facilitate early implementation of treatment strategies, and to allow families to plan for the future. An integrated approach involving both pharmacological and psychosocial strategies is essential for effective care and management. This approach often includes community support services, professional groups and associations, specialized dementia clinics, and geriatric outreach services.

\section{Pharmacologic treatment of dementia}

Two classes of drugs are currently approved for the symptomatic treatment of dementia: acetylcholinesterase inhibitors (AChEI) and $N$-methyl-D-aspartic acid (NMDA) receptor antagonists (Table 3). These pharmacologic treatments do not target the underlying pathogenesis of dementia, but rather help to improve or maintain function following neuronal damage. ${ }^{89}$

The development of AChEIs emerged from a pathophysiologic model of AD known as the amyloid cascade hypothesis. In this model, it is proposed that accumulation of $\mathrm{A} \beta$ in the brain is the primary force driving $\mathrm{AD}$ pathogenesis, with the rest of the disease process resulting from an imbalance between $A \beta$ production and clearance. Within the cascade, a dysregulation in amyloid precursor

Table 3 Pharmacologic treatments for dementia

\begin{tabular}{|c|c|c|c|}
\hline Drug name & Mechanism & $\begin{array}{l}\text { Daily } \\
\text { dosage }\end{array}$ & Side effects \\
\hline Donepezil & AChE inhibitor & $5-10 \mathrm{mg}$ & $\begin{array}{l}\text { Nausea, vomiting, } \\
\text { diarrhea, vivid } \\
\text { dreams, leg cramps }\end{array}$ \\
\hline Rivastigmine & AChE inhibitor & $3-12 \mathrm{mg}$ & $\begin{array}{l}\text { Nausea, vomiting, } \\
\text { diarrhea, weight } \\
\text { loss, anorexia }\end{array}$ \\
\hline Galantamine & AChE inhibitor & $8-12 \mathrm{mg}$ & $\begin{array}{l}\text { Nausea, vomiting, } \\
\text { diarrhea, weight } \\
\text { loss, anorexia }\end{array}$ \\
\hline Memantine & $\begin{array}{l}\text { NMDA receptor } \\
\text { antagonist }\end{array}$ & $10-20 \mathrm{mg}$ & $\begin{array}{l}\text { Hallucinations, } \\
\text { confusion }\end{array}$ \\
\hline
\end{tabular}

Abbreviations: AChE, acetylcholinesterase; NMDA, N-methyl-D-aspartic acid. protein (APP) processing by $\beta$ - and $\gamma$-secretases initiates the pathogenic events leading to aggregation of $A \beta$, specifically $\mathrm{A} \beta 42$, an insoluble fragment that deposits in plaques. The formation of these amyloid plaques further instigates pathological events, including the formation of neurofibrillary tangles (NFTs) from hyperphosphorylation of the tau protein, disruption of synaptic connections, reduction in neurotransmitters, death of tangle-bearing neurons, harmful oxidative processes, damaging inflammatory responses, and the eventual emergence of the clinical symptoms of dementia. ${ }^{90}$ Although the amyloid cascade hypothesis has gained wide acceptance in the field of dementia research, it remains controversial. Emerging evidence shows that many other neuropathological processes may be involved, including the theory that the central mechanism is the hyperphosphorylation of the tau protein. More recent data from multidisciplinary research has suggested the importance of vascular risk factors (eg, smoking, obesity, hypercholesterolemia) and vascular morbidity (eg, hypertension, diabetes, silent brain infarcts, and white matter lesions) in the pathology of dementia. ${ }^{5,91,92}$

\section{Pharmacotherapy for AD}

There are four currently approved AChEIs for the treatment of $\mathrm{AD}$ : tacrine, donepezil, rivastigmine, and galantamine. All of these drugs are approved for mild to moderate $\mathrm{AD}$, with the exception of donepezil, which is approved for mild to severe AD. ${ }^{89}$ Head-to-head clinic trials comparing the effectiveness of each of the AChEIs are rare, thus making it difficult to determine which is best for the treatment of patients with AD. Tacrine has an inconvenient dosing schedule (four doses daily), and significant side effects (hepatotoxicity), and is rarely used in current clinical practice. ${ }^{91}$ The other three AChEIs appear to be relatively equivalent in terms of efficacy and are associated with symptomatic improvement in cognitive function in patients with mild to moderate $\mathrm{AD}$. Improvement generally lasts for 6 to 12 months, followed by a gradual decline in most, but not all, cases ${ }^{89}$

There is some controversy on the duration of use of AChEIs in the treatment of patients with AD. Some clinicians feel that once a patient has reached the stage of severe $\mathrm{AD}$, AChEI use should be discontinued. Others feel that AChEIs may still be useful for managing symptoms in severe $A D$, but this remains an active and open area of clinical investigation. Despite this controversy, AChEIs represent the primary form of treatment for AD.

Memantine is currently the only NMDA antagonist approved to treat $\mathrm{AD}$. The rationale behind the use of 
memantine is the glutamatergic excitotoxicity theory, which stipulates that an abnormal and sustained increase in glutamate may lead to neuronal degeneration. Memantine, an NMDA receptor, is a low-to-moderate uncompetitive antagonist that blocks glutamate binding to its receptor, thereby decreasing neuronal excitotoxicity and slowing the progression of AD. ${ }^{93}$ Memantine has been approved for the treatment of all stages of $\mathrm{AD}$, but in clinical practice is generally used in moderateto-severe AD. Administered twice daily, compliance is a common issue with memantine, as it is difficult for patients with $\mathrm{AD}$ to maintain their dosage schedule. Memantine is a symptomatic treatment for $\mathrm{AD}$, and has been shown to improve cognitive function and slow the rate of decline in moderateto-severe cases. The duration and extent of cognitive efficacy is approximately 6 months, and may be greater. ${ }^{94}$ Additional studies are needed to determine whether the efficacy of memantine in patients with moderate-to-severe AD extends beyond 6 months.

\section{Pharmacotherapy for non-AD dementias}

Vascular dementia can be treated by the use of donepezil, rivastigmine, and galantamine in patients with probable and possible VaD. ${ }^{47}$ Several clinical trials have shown that antihypertensive therapy can help to prevent the onset of $\mathrm{VaD}$ in certain individuals. ${ }^{95}$

DLB is a form of dementia that is not easily treated through the use of pharmacotherapy. Rivastigmine has shown some improvement in DLB patients, and low-dose clonazepam has been used for sleep disturbances. Psychostimulants, including levodopa and dopamine agonists, have been shown to improve cognition, apathy, and psychomotor slowing. Management of cognitive fluctuations in DLB has been difficult. Atypical neuroleptics have also been reported to be helpful for symptoms of delusions and agitation, and selective serotonin reuptake inhibitors (SSRIs) can be effective for the treatment of depression and anxiety in DLB patients. ${ }^{96}$

Frontotemporal dementia is another challenging form of the disease where management and treatment strategies have been inconsistent and disappointing to date. Few large-scale, clinical trials for the treatment of FTD have been conducted, although several smaller trials have been carried out. In these studies, rivastigmine and SSRIs have been shown to improve behavioral symptoms of FTD, but cognitive improvement has been limited. ${ }^{97,98}$

\section{Goals of pharmacotherapy for dementia}

At the initiation of pharmacologic treatment for dementia, the goals of therapy should be clearly established with the patient and their caregivers. AChEIs and memantine are not as effective as drug medications for other medical conditions, and it is important to clarify that they do not alter the eventual course of the disease. The treatment options available mainly stabilize the patient, delaying subsequent decline, with some individuals experiencing transient symptomatic improvements. Clinicians must set realistic goals that take into account the modest benefits that can be expected in various cognitive, behavioral, emotional, and functional domains.

\section{Nonpharmacologic treatment of dementia}

Over the past 3 decades, interest has grown in the use of nonpharmacologic treatment approaches, alone or in combination with pharmacotherapy, for the treatment and management of dementia. Several therapies have been suggested including cognitive-based therapies, psychosocial therapies, physical therapies, and sensorial therapies. These strategies may be implemented in a variety of settings (home, institutional care facility) and treatment modalities (individual or groupbased therapy). The clinical efficacy of nonpharmacologic treatments remains to be fully evaluated, but it is understood that the primary goal of these approaches is to improve the quality of life for individuals with dementia.

\section{Cognitive-based interventions}

Cognitive interventions for dementia rely on the plasticity hypothesis, which theorizes that the brain is able to achieve neural and functional improvements by reorganizing its constituent elements and internal network connectivity according to environmental constraints. ${ }^{99}$

Cognitive training and cognitive rehabilitation are the two most commonly applied nonpharmacologic strategies to implement in the early stages of dementia. These interventions involve targeted practice and training of specific cognitive domains, with a primary emphasis on memory, attention, and executive functions. Cognitive rehabilitation differs from cognitive training in that it is a more individualized therapeutic approach, and its goals are to enhance functioning in everyday life, rather than improve performance on cognitive tasks. A recent Cochrane review examined the efficacy of cognitive training and rehabilitation in patients with early stage $\mathrm{AD}$ and $\mathrm{VaD}$ in five domains from nine randomized controlled trials including participant scores on cognitive screening measures, neuropsychological tests, self-reported functioning, informant ratings of functioning, and reactions to memory impairments and behavioral difficulties. While some positive results were found in specific cognitive 
domains, overall improvements were limited and there were no significant differences between cognitive training and control groups. ${ }^{100}$

\section{Psychosocial therapies}

Psychosocial therapy is an intervention that is intended to enhance self-esteem, well-being, social and communication skills, and to decrease behavioral disturbances.

Reminiscence therapy is a common psychosocial intervention in dementia care. Initially introduced in the 1980s, this approach is based on evocation and discussion between the dementia patient and trusted individuals to think about and revisit past events, experiences, and activities. It often involves the use of objects and supports (eg, photos, personal belongings, music) to help trigger specific memories. Very few randomized controlled trials have assessed reminiscence strategies in dementia. A recent Cochrane meta-analysis concluded that reminiscence therapy resulted in significant improvements in behavioral functioning, as well as cognitive and depressive symptoms, compared with no-treatment and social-contact control groups, with sustained effects 4 to 6 weeks after cessation of the intervention. ${ }^{101}$

Validation therapy is another form of psychosocial intervention for the treatment of dementia. The goal of validation therapy is to promote and stimulate communication skills in the dementia patient, and to provide the individual with insight into their external reality. A recent Cochrane review found little evidence supporting this form of intervention, but noted that the limited information from randomized controlled trials is insufficient to draw reliable conclusions about the efficacy of validation therapy in dementia. ${ }^{102}$

\section{Physical activity therapy}

Physical activity can be used as a therapeutic approach in a wide range of target populations including healthy aging adults and individuals with dementia. The benefits of physical activity have been demonstrated in terms of mood, quality of life, falls, cardiovascular function, and disability rates. ${ }^{103-106}$ Regular exercise can also slow down or help prevent functional decline associated with aging, improve muscle mass, arterial compliance, energy metabolism, cardiovascular fitness, and overall functional capacity. ${ }^{107}$

An individual's overall physical fitness is composed of several components including cardiorespiratory and muscular fitness, flexibility, and balance. Cardiorespiratory fitness, also known as aerobic training, is a condition in which the cardiovascular and respiratory systems function together to ensure that adequate oxygen is supplied to working muscles to produce energy. A high level of cardiorespiratory fitness permits continuous physical activity without a decline in performance and allows for rapid recovery. It is also believed that cardiovascular fitness can improve cognitive capacities. Animal research has demonstrated beneficial effects of aerobic physical activity on cognition, through physiological processes involved in increased cerebral blood flow, oxygen extraction, and glucose utilization. ${ }^{108}$ It has therefore been suggested that physical activity may act as a protective factor for cognitive decline in older adults. ${ }^{109}$

A recent Cochrane review examined the effects of physical activity (aerobic exercise training or physical activity of any length of time) on cognition, function, and behavior in individuals with dementia. The review also evaluated caregiver outcomes and healthcare service use. No significant results were observed but numerous methodological factors limited the potential findings of the meta-analysis. ${ }^{110}$ More studies with homogeneous populations, in terms of dementia subtype and severity, are required to fully investigate the role of physical activity in dementia. Moreover, several key questions about physical activity remain to be clarified, including how long the effects of exercise last after cessation of training, and how much exercise is required to exert a beneficial effect.

\section{Managing and treating BPSD}

BPSD occur in individuals with any of the dementia subtypes and include a heterogeneous range of psychological reactions, psychiatric symptoms, and behavioral disturbances. BPSD can cause serious complications often leading to increased emergency department visits, caregiver distress and illness, increased financial costs to society, early institutionalization, and diminished quality of life. ${ }^{12,13,81,111,112}$ Therefore, effective management of BPSD in dementia is an important treatment consideration.

BPSD can generally be separated into behavioral excesses, wherein the occurrence of the behavior is problematic (eg, physical and verbal aggression, agitation, anxiety, disinhibition, depression, aberrant motor behavior, irritability, hallucinations, and delusions) and behavior deficits, wherein the nonoccurrence of the behavior causes the complications and challenges (eg, apathy leading to withdrawal from social interactions, cessation of self-care including eating and dressing, and sleep disturbances (Table 4). ${ }^{11,113,114}$ The most frequently occurring BPSD are apathy, depression, and anxiety. ${ }^{115,116}$ BPSD can occur at any stage along the dementia continuum, but certain symptoms have been found to be more common in mild dementia (eg, depression, anxiety, 
Table 4 Neuropsychiatric features of behavioral and psychological symptoms of dementia

\begin{tabular}{ll}
\hline Presentation & Clinical features \\
\hline Behavioral and & Aggression: aggressive resistance, verbal \\
psychological excess & or physical aggression \\
& Agitation: aimless walking, pacing, trailing, \\
& restlessness, repetitive actions, sleep \\
& disturbances \\
& Psychosis: hallucinations, delusions, \\
& misidentification \\
Behavioral and & Depression: sad, tearful, hopeless, low \\
psychological deficits & self-esteem, anxiety, guilt \\
& Apathy: withdrawal, lack of motivation, \\
& loss of interest \\
\hline
\end{tabular}

irritability, and apathy), while others occur more frequently in later stages of the disease process (eg, aberrant vocalizations, delusions, hallucinations, and disinhibition). ${ }^{117-119}$ Agitation and aggression are among the most challenging BPSD for caregivers, and along with psychosis and depression are the leading predictors of institutionalization. ${ }^{120}$ When present, inappropriate sexual behaviors have been rated by caregivers as the most difficult symptom of BPSD to manage. ${ }^{121,122}$

A variety of biological, psychosocial, and environmental factors have been proposed to explain the development of BPSD. Psychosocial models of BPSD include: Need-Driven Dementia Compromised Model, Progressively Lowered Stress Threshold Model, and the Biopsychosocial Model of Dementia.

\section{Need-Driven Dementia Compromised Model}

Within the context of the Need-Driven Dementia Compromised Model, BPSD is considered to be the most integrated and meaningful response possible by an individual with dementia to their environment. ${ }^{123}$ According to Algase, BPSD arise due to the pursuit of a goal or as an expression of a need by the individual with dementia. It is also suggested that the presence, severity, and type of BPSD is a reflection of the interaction between salient background factors which are somewhat stable individual characteristics (eg, gender, personality type, general health), and proximal factors which are more fluid or fluctuating aspects of the immediate physical and social environment, as well as the dynamic or changing needs and internal states of the person with dementia (eg, temperature, excessive noise, hunger, fear). Seen this way, the Need-Driven Dementia Compromised Model defines BPSD as meaningful and potentially useful behaviors that can direct and inform appropriate caregiver responses. The primary goal is therefore not to eliminate the behaviors completely, but rather to understand them and enable caregivers to more readily and accurately meet the needs of the person with dementia. ${ }^{123}$

\section{Progressively Lowered Stress Threshold Model}

The Progressively Lowered Stress Threshold Model posits that persons with dementia are increasingly less able to manage stress as the disease progresses. ${ }^{124}$ This reduction in their capacity to tolerate stress results in anxious behaviors as an attempt to alleviate perceived stress. According to Hall and Buckwalter, BPSD (referred to as catastrophic reactions) occur if the stress is unrelieved through either adaptation of the environment or appropriate responses from caregivers or both. ${ }^{124}$

\section{Biopsychosocial model of dementia}

The biopsychosocial model of dementia is a theoretical framework developed to illustrate the role of psychosocial factors within the context of biological processes and to increase understanding of the factors that may lead to improvement or deterioration in dementia. ${ }^{125}$ This model suggests that in both biological and psychosocial domains there are fixed factors, which are not amenable to change (eg, age, education, gender, personality traits), and tractable factors, which are more flexible and adaptive (eg, pain, physical and social environment, level of physical and social activity). The trajectory of dementia is presented as a process, beginning with aging, early organic change, initial cognitive impairments, formal diagnosis of dementia, and then moves progressively towards dependency, institutionalization, end of life care, and eventual death. In the biopsychosocial model, the fixed and the tractable factors will simultaneously influence the point at which the symptoms of dementia begin, the nature and speed of the deterioration, and the effectiveness and appropriateness of interventions used by caregivers to assist persons with dementia. The primary aim of this model is to increase caregivers' ability to identify ways to improve the quality of life and reduce excess disability (often by reducing BPSD) for the person with dementia. ${ }^{125}$

\section{Environmental factors}

Environmental factors that have been found to contribute to the development of BPSD can be divided into two subgroups: the physical environment (eg, architectural features, interior design features, and sensory attributes such as lighting and noise levels) and the social environment (eg, optimizing the mix of residents, social stimulation, and sensory stimulation). Physical environmental factors that are precursors to BPSD include inadequate lighting, confusing surroundings, 
excessive noise/stimulation, and poorly designed care environments. ${ }^{120,126}$ Social environmental factors that have been found to contribute to the presence of BPSD include a lack of daily routine, excessive demands from caregivers, distressing behavior of others, loneliness/boredom, and social isolation. ${ }^{120}$

\section{Person-centered care for BPSD}

Many management and treatment interventions for BPSD are underpinned by a person-centered care approach. An important aspect of the person-centered approach is the therapeutic benefit of positive interpersonal interactions. The conditions that influence positive change include having a therapist who provides unconditional positive regard, is empathetic, and is genuine or congruent within the client-therapist relationship. ${ }^{127}$ This person-centered approach has been adopted and applied to dementia individuals in long-term care settings. ${ }^{128}$ It involves recognizing and identifying the personal preferences and needs of dementia patients as a way to guide caregiving, thus enabling the individualization of care plans and routines. Additionally, a central feature of person-centered care is the recognition that all human life is grounded in relationships, and that individuals with dementia need an enriched social environment that fosters opportunities for personal growth while compensating for their cognitive and functional impairments. ${ }^{128,129}$

The key elements of person-centered care include 1) knowing the person as an individual; 2) providing care that is meaningful to the person in ways that respect their values, preferences, and needs; 3 ) viewing patients as biopsychosocial beings; 4) enabling the development of consistent and trusting caregiving relationships; 5) emphasizing freedom of choice and individually defined risk taking; 6) promoting emotional and physical comfort; and 7) involving patients' family, friends, and social networks in care decisions. ${ }^{129}$

Findings from empirical studies demonstrate that the application of person-centered care results in reduced neuroleptic medication use and agitation. ${ }^{130,131}$ Despite the widespread recognition and application of person-centered care for individuals with dementia, many studies to date are conceptual or anecdotal, highlighting the need for more research on this treatment approach. ${ }^{132}$

\section{Nonpharmacological treatments for BPSD}

Nonpharmacological treatments for BPSD can be grouped into two categories: 1) indirect interventions aimed at decreasing BPSD through indirect work with caregivers or the environment, and 2) direct interventions targeted directly at individuals to decrease BPSD.

\section{Indirect interventions Caregiver training}

The aim of caregiver training is to increase formal and informal caregiver understanding of BPSD, and improve skills in managing and responding to problem behaviors. Education and training programs have been found to be effective in the reduction of BPSD in both nursing home environments, and the community. ${ }^{133,134}$ Multidisciplinary team approaches and individualized treatment plans developed according to the unique needs of the individual with dementia, combined with caregiver problem-solving techniques are key features of successful education and training interventions. ${ }^{135,136}$

\section{Environmental adaptations}

When BPSD appear to be triggered by environmental factors, interventions aimed at modifying the individual's environment may be the most effective means to address the behaviors. Key environmental features include: 1) architectural elements, such as room layout and size (eg, smaller social spaces); 2) interior design features, such as furniture type and arrangement (eg, homelike furnishing, familiar objects, and personalized rooms); and 3) sensory attributes (eg, noise, lighting, and visual accessibility). Adaptations of these environmental features have been found to reduce anxiety, increase a sense of emotional well-being, and enhance social interaction for individuals with dementia. ${ }^{126,132,137-139}$

\section{Direct interventions}

\section{Sensory therapy}

Various sensory-based therapies have been developed for use with dementia populations, including music therapy, light therapy, and Snoezelen therapy. Music therapy is defined as the use of music or musical elements (voice, sound, rhythm) by a qualified music therapist. This nonpharmacologic approach is believed to promote nonverbal communication, relationships, learning, expression, and to improve emotional, social, and cognitive functioning through increased quality of life. Music therapy can be applied on an individual or group level, and may be active or receptive. Several randomized controlled trials of music therapy in dementia have recently been conducted, and significant positive effects have been found in the reduction of BPSD. Specific improvements were found on indices of anxiety and depression for up to 8 weeks after discontinuation of music therapy. ${ }^{140-142}$ Background music has also been shown to be effective in reducing agitation. ${ }^{143,144}$ 
The complexity of BPSD in dementia suggests that a multi-factorial and integrated approach is required for appropriate intervention and management. Approaches that do not take into consideration the complex biological, psychosocial, psychological, and environmental factors will likely produce results that are sporadic, inconsistent and short lived. Consequently, a comprehensive and integrated approach, which addresses the needs of the individual with dementia, the caregivers, and the physical and psychosocial environment is highly recommended.

\section{Empirical research on dementia: toward facilitating early detection of those at risk}

Considerable effort has been devoted to better understanding change in cognitive functioning during the prodromal phase of dementia. ${ }^{145}$ This research focus is well intentioned given the protracted nature of neuropathological change in dementia, coupled with the fact that effective treatment pharmacotherapy remains elusive. ${ }^{146}$ Ultimately, the success of potential interventions will be further augmented through the early detection of those at risk. In this spirit, we briefly summarize some recent empirical findings of preclinical cognitive deficits in $\mathrm{AD}$, and discuss some potentially fruitful avenues for future research.

\section{Characterizing cognitive decline during the prodromal phase of dementia}

A recent meta-analysis examined 47 studies comprising more than $1200 \mathrm{AD}$ cases and 9000 controls. ${ }^{147}$ Among the primary foci, the meta-analysis examined preclinical AD impairment across multiple cognitive domains, as well as the moderating impact of the length of the prodromal period on the magnitude of the preclinical AD effect. Consistent with expectation, larger prodromal deficits were observed for studies characterized by a time interval of less than 3 years between cognitive assessment and clinical diagnosis, relative to studies with average follow-up periods greater than 3 years. For example, episodic memory differences between cases and controls were characterized by a large effect (1.12 SD units) within 3 years of diagnosis, but a moderate effect (0.76 units) for studies with follow-up intervals greater than 3 years between testing and diagnosis. Another critical finding from the meta-analysis concerned the pattern of deficits as a function of cognitive domain. Consistent with the etiology of $\mathrm{AD}$, large magnitude deficits in episodic memory were observed for $\mathrm{AD}$ cases relative to controls.
Large-magnitude cognitive deficits were also observed, however, for measures of executive functioning, processing speed, and global cognition, consistent with the notion that deficits in multiple cognitive domains characterize the prodromal phase of dementia.

On the surface, detecting such large-magnitude deficits would represent seemingly positive news vis-à-vis early detection of those at risk of dementia. However, despite the large magnitude differences in mean performance across multiple cognitive outcomes, considerable overlap $(>40 \%)$ remained across performance distributions for the preclinical $\mathrm{AD}$ vs nondemented groups, with this overlap severely impairing task sensitivity for detecting those at risk of dementing. ${ }^{147}$ Further to the point, it is arguably unrealistic to expect nonoverlapping distributions of cognitive performance many years before diagnosis given the large interindividual variability in both preclinical AD cases, and those who will remain without dementia. ${ }^{148-150}$ Numerous factors can result in cognitive impairment with increasing age, leading to false-positive detection including psychiatric, metabolic, immunological, and circulatory conditions. ${ }^{151-154}$ Moreover, some individuals who will convert to dementia show accelerated decline only in close proximity to diagnosis, leading to false negatives. ${ }^{149}$

\section{Change-point models of onset and rate of cognitive decline before dementia diagnosis}

Given the problem of overlapping distributions for groups of preclinical $\mathrm{AD}$ and nondemented controls, an obvious question concerns what can be done to improve classification of those at risk? Based on recent findings from our research group, we argue that facilitating early disease identification and targeting successful intervention requires moving beyond static mean-difference comparisons at one point in time to consideration of the relative onset and rate of cognitive decline across a continuum of cognitive measures. ${ }^{155}$ To examine these issues, Thorvaldsson and colleagues employed data from two population-based Swedish longitudinal studies of aging. ${ }^{155}$ Participants from the age-heterogeneous Kungsholmen Project (Stockholm, Sweden) were aged 75 years or older and completed up to 5 measurement occasions (417 dementia cases and 249 noncases). The Gerontological and Geriatric Population Studies (Gothenburg, Sweden) included participants who were all initially 70 years of age and who were followed for up to 13 retest occasions spanning 30 years (113 dementia cases and 272 noncases). A series of linear mixed change-point models (see Hall, Lipton, Sliwinski, and Stewart, 2000 ${ }^{156}$ ) were fit 
to numerous cognitive outcomes. These piecewise models estimated two slopes, permitting the knot of the spline (the change point) to vary across models in 1-month increments. Prior to the knot, time was parameterized as a function of chronological age (years since birth from each individual measurement). In contrast, following the knot, time was specified as a function of time to dementia diagnosis (years to diagnosis from each individual measurement). A profile likelihood method was then employed to select the best fitting change point spanning a range from 1 month to 10 years prior to diagnosis (eg, 3.1 years before diagnosis, 3.2 years before diagnosis, 3.3 years before diagnosis). These systematic model fit comparisons facilitated the identification of the average transition point that best differentiated between normative age-related cognitive decline and accelerated decline during the preclinical phase.

Thorvaldsson et al reported that the earliest preclinical deficits, around 9 years before diagnosis, were observed for cognitive correlates of dementia pathology (episodic memory) as well as more executively demanding tasks (eg, visuospatial ability, processing speed). ${ }^{155}$ Notably, however, as nondemented elderly individuals also exhibited decline on these tasks, the relative magnitude of cognitive changes for the preclinical cases was only modestly incremented. Consistent with expectation, tasks that exhibited the earliest onset of cognitive decline did not also exhibit the fastest rate of change. Rather, the most precipitous estimates of decline were observed for tasks that are at least somewhat resistant to age-related decline such as category fluency - a knowledge-based task that incorporates both fluid and crystallized abilities. Such patterns suggest that tasks incorporating both fluid and well-preserved crystallized abilities may facilitate discrimination between preclinical cases vs noncases, but only in close proximity to incident diagnosis.

\section{Onset versus rate of change}

In light of these findings underscoring that onset and rate of change in the prodromal period vary as a function of cognitive domain, another critical question concerns which characteristic to focus on for increasing sensitivity of detecting those at risk of dementing. As with most questions, the answer depends on whether an individual's emphasis concerns clinical practice or basic research. A general practitioner interested in the health of an individual patient should place more emphasis on early detection, and thus differentially focus on onset. Based on our findings, this would mean targeting early behavioral changes in process-based measures of cognition (eg, episodic memory) that exhibit impairments up to 10 or more years prior to diagnosis. Of course, clinical detection of such onset in change requires knowledge of baseline performance and repeated within-person assessments, and even then may prove difficult to detect as normative agerelated changes are also typically observed for these same measures. In the Thorvaldsson et al study, for example, episodic memory decline in the prodromal phase was only accelerated by a factor of 2 relative to decline observed during normal aging. ${ }^{155}$

In contrast, those focusing on more basic research (eg, targeting individuals at greatest-risk of converting to $\mathrm{AD}$ for use in clinical trials research, identifying candidates for aggressive pharmacological intervention) may differentially focus on accelerated rate of change (rather than onset) for select cognitive domains. In particular, our findings suggest that verbal fluency may be a particularly important measure for increasing sensitivity of detection for those at risk of dementing. Verbal fluency shows very little age-related change, but when change is finally manifest during the prodromal period within 6 years of diagnosis, performance decline accelerates by a factor of 5 or more relative to normal aging. Indeed, clinical trials research might consider adopting a multivariate approach where at-risk participants are selected on the basis of both onset (individuals exhibit early change in episodic memory consistent with AD etiology) as well as change (individuals with episodic deficit who also show accelerated decline in verbal fluency); such an approach is likely to improve sensitivity of identifying those at greatest risk of converting.

\section{Preclinical cognitive deficits and threshold models}

Fundamental tenets of threshold models of cognitive impairment are consistent with both the Thorvaldsson et al findings, demonstrating inflection points that differentiate between normative age-related cognitive decline vs more accelerated decline during the prodromal period in proximity to diagnosis, and the Bäckman et al meta-analysis demonstrating that cognitive function is more strongly affected in proximity to diagnosis. ${ }^{147,155}$ Further, the concepts of cognitive reserve as well as compression of morbidity are both consistent with initial trajectories of change that are gradual, followed by more pronounced cognitive decline in proximity to diagnosis. ${ }^{157-159}$ For example, Stern's notion of cognitive reserve was developed to facilitate explanation on the modest association between the amount of underlying AD pathology and observed behavioral deficit. ${ }^{159}$ Cognitive reserve is said 
to reflect the amount of neuropathology that can be accrued prior to reaching a critical threshold beyond which cognitive deficits are manifest. ${ }^{159}$ Educational attainment in particular has been viewed as an index of cognitive reserve, with recent findings clearly demonstrating the aforementioned threshold effects. Hall et al, for example, showed that those with more years of education $(12+)$ did not exhibit cognitive decline onset until close proximity to dementia diagnosis relative to a low-educated group (less than 7 years) who would subsequently dement. ${ }^{157}$ However, upon reaching their threshold for pathology burden, the rate of cognitive impairment was steeper for the high-educated group. Thus, later disease onset appears to be associated with greater disease burden, manifest as accelerated cognitive loss (ie, steeper prodromal slopes) once decline occurs.

\section{Future research}

In light of the meta-analytic findings by Bäckman et al, it is apparent that prospective identification is not facilitated through solitary use of cognitive measures that exhibit large mean differences between dementia cases and controls years before diagnosis. ${ }^{147}$ This is because the distributions between the two groups still overlap to a large extent $(>40 \%)$ for individual cognitive domains such as episodic memory or executive functioning. To increase sensitivity of early detection, Small et al suggest that future research incorporates the following improvements. ${ }^{145}$ First, these authors emphasize that multifactorial prediction models are required to improve early detection. Evidence suggests that early detection is improved considerably upon inclusion not only of episodic memory tasks, but also indicators of executive functioning and semantic memory. ${ }^{160,161}$ Next, in service of better differentiating between cases and controls, future research should also supplement such cognitive markers with other markers linked to dementia incidence. These multivariate models and their corresponding interactions could include biomarkers such as brain volumetrics, amyloid deposition, and white-matter alterations, as well as genetic markers, subjective memory complaints, social isolation, and lifestyle factors such as lack of intellectual engagement or low physical activity levels. ${ }^{149,162-169}$ A key challenge for future research will be to ascertain whether specific combinations of these biological, social, cognitive, genetic, and clinical markers increase identification of those at risk of dementia. To the extent that protective characteristics (eg, high education, active lifestyle) mask the early signs of AD and delay the clinical onset, effective multivariate (multistage) approaches for facilitating detection will be essential given the reduced time window between onset of preclinical decline and subsequent diagnosis. ${ }^{170}$ However, an alternate view is that delaying the clinical manifestation of AD by just 2 years could significantly reduce the total number of AD cases as well as helping to lower healthcare costs. ${ }^{171}$ This latter view underscores the critical need to identify sensitive early indicators of those at risk, and then to target interventions such as health or behavior promotion strategies (eg, physical or cognitive lifestyle interventions), which may successfully delay (or prevent) onset of dementia. Finally, given the importance of understanding the natural history of AD progression, more sophisticated longitudinal designs should be adopted for the study of cognitive change during the preclinical period through to dementia onset. In particular, including multiple measurement occasions before diagnosis is required to better identify the onset of cognitive decline during the prodromal period, as well as to quantify the magnitude of acceleration. Multiwave datasets and linear mixed models may also identify whether select dementia correlates increase or decrease in importance in proximity to diagnosis. ${ }^{145}$ Such differentiation may help differentiate between disease risk factors vs precipitating factors.

\section{Summary: toward a paradigm shift in dementia care for the 2 I st century}

The preceding review highlights the many advances over the past few decades that aid our understanding of dementia epidemiology and its clinical presentation. It outlines various multidisciplinary treatments, including pharmacological and nonpharmacological treatment, and addresses significant management challenges. Demographic projections underscore the potential burden that the various dementias pose for healthcare in the coming 20 years. Dementia prevalence will continue to increase in concert with increased life expectancy and improved medical care. Unequivocally, clear advances have been made in dementia diagnosis, identification of associated risk factors, as well as management and care. However, effective pharmacological treatments that target the underlying neuropathology and alter the disease course remain elusive.

At least in the near term, we can argue that dementia is as much about social policy and governance as it is about medicine or neuropsychology. Given the progressive nature of the disease, it will be necessary to target preventive as opposed to reactive interventions. Improved policy initiatives should target public education documenting specific risk factors for dementia from early- and mid-life, as well as lifestyle interventions (eg, health or behavior promotion strategies) 
that can potentially delay or prevent the disease. In the United States, for example, delaying the clinical manifestation of dementia by 2 years would reduce the total number of AD cases by approximately $600,000 .{ }^{171}$ Based on increasing prevalence projections in Canada, the Alzheimer Society of Canada (2010) Rising Tide report projects the total economic burden of dementia to double each decade, increasing from approximately $\$ 15$ billion (CAD) per year in 2008 to $\$ 153$ billion (CAD) per year by $2038 .{ }^{172}$ Clearly, delaying the onset of dementia by even several years would have considerable impact on both disease prevalence as well as associated healthcare expenditures. To realize such benefits, policy makers should place continued emphasis on dementia research, as well as renewed focus on public education initiatives (eg, increased as opposed to decreased emphasis on physical education programs in primary and secondary school) that promote protective and enduring lifestyle behaviors.

\section{Acknowledgments}

Jacob Grand was supported by a Frederick Banting and Charles Best Doctoral Research fellowship from the Canadian Institutes for Health Research (CIHR). Sienna Caspar acknowledges support by a Joseph-Armand Bombardier Canada Graduate Scholarships Program from the Social Sciences and Humanities Research Council. Stuart MacDonald was supported by a Career Investigator Scholar Award from the Michael Smith Foundation for Health Research (MSFHR).

\section{Disclosure}

The authors declare no conflicts of interest.

\section{References}

1. From the Centers for Disease Control and Prevention. Public health and aging: trends in aging - United States and worldwide. JAMA. Mar 19 2003;289(11):1371-1373.

2. Wimo A, Winblad B, Aguero-Torres H, von Strauss E. The magnitude of dementia occurrence in the world. Alzheimer Dis Assoc Disord. Apr-Jun 2003;17(2):63-67.

3. Ferri CP, Prince M, Brayne C, et al. Global prevalence of dementia: a Delphi consensus study. Lancet. Dec 17 2005;366(9503):2112-2117.

4. Lobo A, Launer LJ, Fratiglioni L, et al. Prevalence of dementia and major subtypes in Europe: A collaborative study of population-based cohorts. Neurologic Diseases in the Elderly Research Group. Neurology. 2000; 54(11 Suppl 5):S4-S9.

5. Qiu C, Kivipelto M, von Strauss E. Epidemiology of Alzheimer's disease: occurrence, determinants, and strategies toward intervention. Dialogues Clin Neurosci. 2009;11(2):111-128.

6. Brookmeyer R, Johnson E, Ziegler-Graham K, Arrighi HM. Forecasting the global burden of Alzheimer's disease. Alzheimers Dement. Jul 2007;3(3):186-191.

7. Alva G, Potkin SG. Alzheimer disease and other dementias. Clin Geriatr Med. Nov 2003;19(4):763-776.
8. Geldmacher DS. Differential diagnosis of dementia syndromes. Clin Geriatr Med. Feb 2004;20(1):27-43.

9. Manning C. Beyond memory: neuropsychologic features in differential diagnosis of dementia. Clin Geriatr Med. Feb 2004;20(1):45-58.

10. Kester MI, Scheltens P. Dementia: the bare essentials. Pract Neurol. Aug 2009;9(4):241-251.

11. Mirakhur A, Craig D, Hart DJ, McLlroy SP, Passmore AP. Behavioural and psychological syndromes in Alzheimer's disease. Int J Geriatr Psychiatry. Nov 2004;19(11):1035-1039.

12. Steinberg M, Corcoran C, Tschanz JT, et al. Risk factors for neuropsychiatric symptoms in dementia: the Cache County Study. Int J Geriatr Psychiatry. Sep 2006;21(9):824-830.

13. Lyketsos CG, Lopez O, Jones B, Fitzpatrick AL, Breitner J, DeKosky S. Prevalence of neuropsychiatric symptoms in dementia and mild cognitive impairment: results from the cardiovascular health study. JAMA. Sep 25 2002;288(12):1475-1483.

14. Grossman H, Bergmann C, Parker S. Dementia: a brief review. Mt Sinai J Med. Nov 2006;73(7):985-992.

15. Crooks EA, Geldmacher DS. Interdisciplinary approaches to Alzheimer's disease management. Clin Geriatr Med. Feb 2004;20(1): 121-139.

16. Camicioli R. Distinguishing Different Dementias. Canadian Review of Alzheimer's Disease and Other Dementias. 2006;9(3):4-11.

17. Petersen RC, Stevens JC, Ganguli M, Tangalos EG, Cummings JL, DeKosky ST. Practice parameter: early detection of dementia: mild cognitive impairment (an evidence-based review). Report of the Quality Standards Subcommittee of the American Academy of Neurology. Neurology. May 8 2001;56(9):1133-1142.

18. Brooks LG, Loewenstein DA. Assessing the progression of mild cognitive impairment to Alzheimer's disease: current trends and future directions. Alzheimers Res Ther. 2010;2(5):28.

19. Palmer K, Wang HX, Backman L, Winblad B, Fratiglioni L. Differential evolution of cognitive impairment in nondemented older persons: results from the Kungsholmen Project. Am J Psychiatry. Mar 2002; 159(3):436-442.

20. Winblad B, Palmer K, Kivipelto M, et al. Mild cognitive impairment beyond controversies, towards a consensus: report of the International Working Group on Mild Cognitive Impairment. J Intern Med. Sep 2004;256(3):240-246.

21. Forlenza OV, Chiu E. Mild cognitive impairment: a concept ready to move on? Curr Opin Psychiatry. Nov 2008;21(6):529-532.

22. Forlenza OV, Diniz BS, Gattaz WF. Diagnosis and biomarkers of predementia in Alzheimer's disease. BMC Med. Dec 22 2010;8(1):89.

23. Forlenza OV, Diniz BS, Nunes PV, Memoria CM, Yassuda MS, Gattaz WF. Diagnostic transitions in mild cognitive impairment subtypes. Int Psychogeriatr. Dec 2009;21(6):1088-1095.

24. Haroutunian V, Hoffman LB, Beeri MS. Is there a neuropathology difference between mild cognitive impairment and dementia? Dialogues Clin Neurosci. 2009;11(2):171-179.

25. Feldman HH, Jacova C. Mild cognitive impairment. Am J Geriatr Psychiatry. Aug 2005;13(8):645-655.

26. Feldman H, Levy AR, Hsiung GY, et al. A Canadian cohort study of cognitive impairment and related dementias (ACCORD): study methods and baseline results. Neuroepidemiology. Sep-Oct 2003;22(5):265-274.

27. Hsiung GY, Donald A, Grand J, et al. Outcomes of cognitively impaired not demented at 2 years in the Canadian Cohort Study of Cognitive Impairment and Related Dementias. Dement Geriatr Cogn Disord. 2006;22(5-6):413-420.

28. Minati L, Edginton T, Bruzzone MG, Giaccone G. Current concepts in Alzheimer's disease: a multidisciplinary review. Am J Alzheimers Dis Other Demen. Apr-May 2009;24(2):95-121.

29. Ready RE, Ott BR, Grace J, Cahn-Weiner DA. Apathy and executive dysfunction in mild cognitive impairment and Alzheimer disease. Am J Geriatr Psychiatry. Mar-Apr 2003;11(2):222-228.

30. Arnaiz E, Almkvist O. Neuropsychological features of mild cognitive impairment and preclinical Alzheimer's disease. Acta Neurol Scand Suppl. 2003;179:34-41. 
31. Nelson AP, O'Connor MG. Mild cognitive impairment: a neuropsychological perspective. CNS Spectr. Jan 2008;13(1):56-64.

32. Griffith HR, Belue K, Sicola A, et al. Impaired financial abilities in mild cognitive impairment: a direct assessment approach. Neurology. Feb 11 2003;60(3):449-457.

33. Plassman BL, Williams JW Jr, Burke JR, Holsinger T, Benjamin S. Systematic review: factors associated with risk for and possible prevention of cognitive decline in later life. Ann Intern Med. Aug 3 2010; 153(3):182-193.

34. Feldman HH, Woodward M. The staging and assessment of moderate to severe Alzheimer disease. Neurology. 2005;65(Suppl 3): S10-S17.

35. Dubois B, Feldman HH, Jacova C, et al. Research criteria for the diagnosis of Alzheimer's disease: revising the NINCDS-ADRDA criteria. Lancet Neurol. Aug 2007;6(8):734-746.

36. Tallberg IM, Almkvist O. Confabulation and memory in patients with Alzheimer's disease. J Clin Exp Neuropsychol. Apr 2001;23(2): $172-184$.

37. Jacova C, Kertesz A, Blair M, Fisk JD, Feldman HH. Neuropsychological testing and assessment for dementia. Alzheimers Dement. Oct 2007;3(4):299-317.

38. Cerhan JH, Ivnik RJ, Smith GE, Tangalos EC, Petersen RC, Boeve BF. Diagnostic utility of letter fluency, category fluency, and fluency difference scores in Alzheimer's disease. Clin Neuropsychol. Feb 2002;16(1):35-42.

39. Vliet EC, Manly J, Tang MX, Marder K, Bell K, Stern Y. The neuropsychological profiles of mild Alzheimer's disease and questionable dementia as compared to age-related cognitive decline. J Int Neuropsychol Soc. Jul 2003;9(5):720-732.

40. Lyketsos CG, Lee HB. Diagnosis and treatment of depression in Alzheimer's disease. A practical update for the clinician. Dement Geriatr Cogn Disord. 2004;17(1-2):55-64.

41. Farcnik K, Persyko MS. Assessment, measures and approaches to easing caregiver burden in Alzheimer's disease. Drugs Aging. 2002; 19(3):203-215.

42. Amano N, Inuzuka S, Ogihara T. Behavioral and psychological symptoms of dementia and medical treatment. Psychogeriatrics. Jun 2009;9(2):45-49.

43. Association AP. Diagnostic and statistical manual of mental disorders. Revised 4th ed. Washington, DC; 2000.

44. McKhann G, Drachman D, Folstein M, Katzman R, Price D, Stadlan EM. Clinical diagnosis of Alzheimer's disease: report of the NINCDSADRDA Work Group under the auspices of Department of Health and Human Services Task Force on Alzheimer's Disease. Neurology. Jul 1984;34(7):939-944.

45. Galasko D, Hansen LA, Katzman R, et al. Clinical-neuropathological correlations in Alzheimer's disease and related dementias. Arch Neurol. Sep 1994;51(9):888-895.

46. Storey E, Slavin MJ, Kinsella GJ. Patterns of cognitive impairment in Alzheimer's disease: assessment and differential diagnosis. Front Biosci. May 1 2002;7:e155-e184

47. Roman GC, Tatemichi TK, Erkinjuntti T, et al. Vascular dementia: diagnostic criteria for research studies. Report of the NINDS-AIREN International Workshop. Neurology. Feb 1993;43(2):250-260.

48. Kalaria RN, Maestre GE, Arizaga R, et al. Alzheimer's disease and vascular dementia in developing countries: prevalence, management, and risk factors. Lancet Neurol. Sep 2008;7(9):812-826.

49. Savva GM, Stephan BC. Epidemiological studies of the effect of stroke on incident dementia: a systematic review. Stroke. Jan 2010;41(1): e41-e46.

50. Pinkston JB, Alekseeva N, Gonzalez Toledo E. Stroke and dementia. Neurol Res. Oct 2009;31(8):824-831.

51. Potluri R, Natalwala A, Uppal H, Heun R. Different risk factors in vascular dementia and ischaemic stroke. Neuroepidemiology. 2009; 32(1):80.

52. Sharp SI, Aarsland D, Day S, Sonnesyn H, Ballard C. Hypertension is a potential risk factor for vascular dementia: systematic review. Int $J$ Geriatr Psychiatry. Dec 292010.
53. Duron E, Hanon O. Hypertension, cognitive decline and dementia. Arch Cardiovasc Dis. Mar 2008;101(3):181-189.

54. DeCarli C. The role of cerebrovascular disease in dementia. Neurologist. May 2003;9(3):123-136.

55. Black S, Gao F, Bilbao J. Understanding white matter disease: imagingpathological correlations in vascular cognitive impairment. Stroke. Mar 2009;40(3 Suppl):S48-S52.

56. Englund E. Neuropathology of white matter lesions in vascular cognitive impairment. Cerebrovasc Dis. 2002;13 Suppl 2:11-15.

57. Kearney-Schwartz A, Rossignol P, Bracard S, et al. Vascular structure and function is correlated to cognitive performance and white matter hyperintensities in older hypertensive patients with subjective memory complaints. Stroke. Apr 2009;40(4):1229-1236.

58. Aggarwal NT, Decarli C. Vascular dementia: emerging trends. Semin Neurol. Feb 2007;27(1):66-77.

59. Roman GC. Vascular dementia may be the most common form of dementia in the elderly. J Neurol Sci. Nov 15 2002;203-204:7-10.

60. Roman GC. Defining dementia: clinical criteria for the diagnosis of vascular dementia. Acta Neurol Scand Suppl. 2002;178:6-9.

61. Chui HC. Subcortical ischemic vascular dementia. Neurol Clin. Aug 2007;25(3):717-740, vi.

62. Benisty S, Hernandez K, Viswanathan A, et al. Diagnostic criteria of vascular dementia in CADASIL. Stroke. Mar 2008;39(3):838-844.

63. Hachinski V. Preventable senility: a call for action against the vascular dementias. Lancet. Sep 12 1992;340(8820):645-648.

64. Small GW. Revised Ischemic Score for diagnosing multi-infarct dementia. J Clin Psychiatry. Dec 1985;46(12):514-517.

65. Organization WH. ICD-10 Classifications of Mental and Behavioral Disorder: Clinical Descriptions and Diagnostic Guidelines. Geneva: World Health Organization; 1992.

66. Snowden J, Neary D, Mann D. Frontotemporal lobar degeneration: clinical and pathological relationships. Acta Neuropathol. Jul 2007; 114(1):31-38.

67. Neary D, Snowden JS, Gustafson L, et al. Frontotemporal lobar degeneration: a consensus on clinical diagnostic criteria. Neurology. Dec 1998;51(6):1546-1554

68. McKhann GM, Albert MS, Grossman M, Miller B, Dickson D, Trojanowski JQ. Clinical and pathological diagnosis of frontotemporal dementia: report of the Work Group on Frontotemporal Dementia and Pick's Disease. Arch Neurol. Nov 2001;58(11):1803-1809.

69. Clinical and neuropathological criteria for frontotemporal dementia. The Lund and Manchester Groups. J Neurol Neurosurg Psychiatry. Apr 1994;57(4):416-418.

70. Arvanitakis Z. Update on frontotemporal dementia. Neurologist. Jan 2010;16(1):16-22.

71. Piguet O, Brooks WS, Halliday GM, et al. Similar early clinical presentations in familial and non-familial frontotemporal dementia. J Neurol Neurosurg Psychiatry. Dec 2004;75(12):1743-1745.

72. Ratnavalli E, Brayne C, Dawson K, Hodges JR. The prevalence of frontotemporal dementia. Neurology. Jun 11 2002;58(11):1615-1621.

73. Graff-Radford NR, Woodruff BK. Frontotemporal dementia. Semin Neurol. Feb 2007;27(1):48-57.

74. McKeith I. Dementia with Lewy bodies. Handb Clin Neurol. 2007;84:531-548.

75. Tiraboschi P, Salmon DP, Hansen LA, Hofstetter RC, Thal LJ, Corey-Bloom J. What best differentiates Lewy body from Alzheimer's disease in early-stage dementia? Brain. Mar 2006;129(Pt 3):729-735.

76. McKeith IG, Dickson DW, Lowe J, et al. Diagnosis and management of dementia with Lewy bodies: third report of the DLB Consortium. Neurology. Dec 27 2005;65(12):1863-1872.

77. Harding AJ, Broe GA, Halliday GM. Visual hallucinations in Lewy body disease relate to Lewy bodies in the temporal lobe. Brain. Feb 2002;125(Pt 2):391-403.

78. Ferman TJ, Boeve BF, Smith GE, et al. Dementia with Lewy bodies may present as dementia and REM sleep behavior disorder without parkinsonism or hallucinations. J Int Neuropsychol Soc. Nov 2002;8(7):907-914. 
79. Verhey FR, Jolles J, Ponds RW, et al. Diagnosing dementia: a comparison between a monodisciplinary and a multidisciplinary approach. $J$ Neuropsychiatry Clin Neurosci. Winter 1993;5(1):78-85.

80. Keough J, Huebner RA. Treating dementia: the complementing team approach of occupational therapy and psychology. J Psychol. Jul 2000;134(4):375-391.

81. Colerick EJ, George LK. Predictors of institutionalization among caregivers of patients with Alzheimer's disease. J Am Geriatr Soc. Jul 1986;34(7):493-498.

82. Gaugler JE, Kane RL, Kane RA, Newcomer R. Early community-based service utilization and its effects on institutionalization in dementia caregiving. Gerontologist. Apr 2005;45(2):177-185.

83. Caron CD, Ducharme F, Griffith J. Deciding on institutionalization for a relative with dementia: the most difficult decision for caregivers Can J Aging. Summer 2006;25(2):193-205.

84. Acton GJ, Winter MA. Interventions for family members caring for an elder with dementia. Annu Rev Nurs Res. 2002;20:149-179.

85. Aminzadeh F, Dalziel WB, Molnar FJ, Garcia LJ. Symbolic meaning of relocation to a residential care facility for persons with dementia Aging Ment Health. May 2009;13(3):487-496.

86. Cutchin MP. The process of mediated aging-in-place: a theoretically and empirically based model. Soc Sci Med. Sep 2003;57(6):1077-1090.

87. Gitlin LN. Conducting research on home environments: lessons learned and new directions. Gerontologist. Oct 2003;43(5):628-637.

88. Buhr GT, Kuchibhatla M, Clipp EC. Caregivers' reasons for nursing home placement: clues for improving discussions with families prior to the transition. Gerontologist. Feb 2006;46(1):52-61.

89. Birks J. Cholinesterase inhibitors for Alzheimer's disease. Cochrane Database Syst Rev. 2006;1:CD005593.

90. Hardy JA, Higgins GA. Alzheimer's disease: the amyloid cascade hypothesis. Science. Apr 10 1992;256(5054):184-185.

91. Saddichha S, Pandey V. Alzheimer's and non-alzheimer's dementia: a critical review of pharmacological and nonpharmacological strategies. Am J Alzheimers Dis Other Demen. Apr-May 2008;23(2):150-161.

92. Querfurth HW, LaFerla FM. Alzheimer's disease. $N$ Engl J Med. Jan 28 2010;362(4):329-344.

93. Rogawski MA, Wenk GL. The neuropharmacological basis for the use of memantine in the treatment of Alzheimer's disease. CNS Drug Rev. Fall 2003;9(3):275-308

94. Reisberg B, Doody R, Stoffler A, Schmitt F, Ferris S, Mobius HJ. Memantine in moderate-to-severe Alzheimer's disease. N Engl J Med. Apr 3 2003;348(14):1333-1341.

95. In’t Veld BA, Ruitenberg A, Hofman A, Stricker BH, Breteler MM. Antihypertensive drugs and incidence of dementia: the Rotterdam Study. Neurobiol Aging. May-Jun 2001;22(3):407-412.

96. Josif S, Graham K. Diagnosis and treatment of dementia with Lewy bodies. JAAPA. May 2008;21(5):22-26.

97. Perry RJ, Miller BL. Behavior and treatment in frontotemporal dementia. Neurology. Jun 2001;56(11 Suppl 4):S46-S51.

98. Boxer AL, Boeve BF. Frontotemporal dementia treatment: current symptomatic therapies and implications of recent genetic, biochemical, and neuroimaging studies. Alzheimer Dis Assoc Disord. Oct-Dec 2007;21(4):S79-S87.

99. Lovden M, Backman L, Lindenberger U, Schaefer S, Schmiedek F. A theoretical framework for the study of adult cognitive plasticity. Psychol Bull. Jul 2010;136(4):659-676.

100. Clare L, Woods RT, Moniz Cook ED, Orrell M, Spector A. Cognitive rehabilitation and cognitive training for early-stage Alzheimer's disease and vascular dementia. Cochrane Database Syst Rev. 2003;4:CD003260.

101. Woods B, Spector A, Jones C, Orrell M, Davies S. Reminiscence therapy for dementia. Cochrane Database Syst Rev. 2005;2:CD001120.

102. Neal M, Briggs M. Validation therapy for dementia. Cochrane Database Syst Rev. 2003;3:CD001394.

103. Netz Y, Wu MJ, Becker BJ, Tenenbaum G. Physical activity and psychological well-being in advanced age: a meta-analysis of intervention studies. Psychol Aging. Jun 2005;20(2):272-284.
104. Spirduso WW, Cronin DL. Exercise dose-response effects on quality of life and independent living in older adults. Med Sci Sports Exerc. Jun 2001;33(6 Suppl):S598-S608; discussion S609-S510.

105. Chang JT, Morton SC, Rubenstein LZ, et al. Interventions for the prevention of falls in older adults: systematic review and meta-analysis of randomised clinical trials. BMJ. Mar 20 2004;328(7441):680.

106. Keysor JJ. Does late-life physical activity or exercise prevent or minimize disablement? A critical review of the scientific evidence. Am J Prev Med. Oct 2003;25(3 Suppl 2):129-136.

107. Lemura LM, von Duvillard SP, Mookerjee S. The effects of physical training of functional capacity in adults. Ages 46 to 90 : a meta-analysis. J Sports Med Phys Fitness. Mar 2000;40(1):1-10.

108. Churchill JD, Galvez R, Colcombe S, Swain RA, Kramer AF, Greenough WT. Exercise, experience and the aging brain. Neurobiol Aging. Sep-Oct 2002;23(5):941-955.

109. Swain RA, Harris AB, Wiener EC, et al. Prolonged exercise induces angiogenesis and increases cerebral blood volume in primary motor cortex of the rat. Neuroscience. 2003;117(4):1037-1046.

110. Forbes D, Forbes S, Morgan DG, Markle-Reid M, Wood J, Culum I. Physical activity programs for persons with dementia. Cochrane Database Syst Rev. 2008;3:CD006489.

111. Schreinzer D, Ballaban T, Brannath W, et al. Components of behavioral pathology in dementia. Int $J$ Geriatr Psychiatry. Feb 2005;20(2):137-145

112. Edell WS, Tunis SL. Antipsychotic treatment of behavioral and psychological symptoms of dementia in geropsychiatric inpatients. Am J Geriatr Psychiatry. Summer 2001;9(3):289-297.

113. De Medeiros K, Robert P, Gauthier S, et al. The Neuropsychiatric Inventory-Clinician rating scale (NPI-C): reliability and validity of a revised assessment of neuropsychiatric symptoms in dementia. Int Psychogeriatr. Sep 2010;22(6):984-994.

114. Vilalta-Franch J, Lopez-Pousa S, Turon-Estrada A, et al. Syndromic association of behavioral and psychological symptoms of dementia in Alzheimer disease and patient classification. Am J Geriatr Psychiatry. May 2010;18(5):421-432.

115. Robert PH, Verhey FR, Byrne EJ, et al. Grouping for behavioral and psychological symptoms in dementia: clinical and biological aspects. Consensus paper of the European Alzheimer disease consortium. Eur Psychiatry. Nov 2005;20(7):490-496.

116. Steinberg M, Shao H, Zandi P, et al. Point and 5-year period prevalence of neuropsychiatric symptoms in dementia: the Cache County Study. Int J Geriatr Psychiatry. Feb 2008;23(2):170-177.

117. Feldman H, Scheltens P, Scarpini E, et al. Behavioral symptoms in mild cognitive impairment. Neurology. Apr 13 2004;62(7) 1199-1201.

118. Lopez OL, Becker JT, Sweet RA, et al. Psychiatric symptoms vary with the severity of dementia in probable Alzheimer's disease. J Neuropsychiatry Clin Neurosci. Summer 2003;15(3):346-353.

119. Finkel SI. Behavioral and psychologic symptoms of dementia. Clin Geriatr Med. Nov 2003;19(4):799-824.

120. Gauthier S, Cummings J, Ballard C, et al. Management of behavioral problems in Alzheimer's disease. Int Psychogeriatr. May 2010;22(3): 346-372.

121. Onishi J, Suzuki Y, Umegaki H, et al. Behavioral, psychological and physical symptoms in group homes for older adults with dementia. Int Psychogeriatr. Mar 2006;18(1):75-86.

122. Tucker I. Management of inappropriate sexual behaviors in dementia: a literature review. Int Psychogeriatr. Aug 2010;22(5):683-692.

123. Algase DL, Beck D, Kolanowski A, Whall A, Berent S, Richards K, et al. Need-driven dementia-compromised behavior: an alternative view of disruptive behavior. Am J Alzheimers Dis. 1996;11(6):10-19.

124. Hall GR, Buckwalter KC. Progressively lowered stress threshold: a conceptual model for care of adults with Alzheimer's disease. Arch Psychiatr Nurs. Dec 1987;1(6):399-406.

125. Spector A, Orrell M. Using a biopsychosocial model of dementia as a tool to guide clinical practice. Int Psychogeriatr. Sep 2010;22(6): 957-965. 
126. Kane RA. Long-term care and a good quality of life: bringing them closer together. Gerontologist. Jun 2001;41(3):293-304.

127. Kirschenbaum H. Carl Rogers' Life and Work: An Assessment on the 100th Anniversary of His Birth. Journal of Counseling and Development. 2004;82(1):116-124.

128. Boise L, White D. The family's role in person-centered care: practice considerations. J Psychosoc Nurs Ment Health Serv. May 2004;42(5):12-20.

129. Talerico KA, O'Brien JA, Swafford KL. Person-centered care. An important approach for 21 st century health care. J Psychosoc Nurs Ment Health Serv. Nov 2003;41(11):12-16.

130. Fossey J, Ballard C, Juszczak E, et al. Effect of enhanced psychosocial care on antipsychotic use in nursing home residents with severe dementia: cluster randomised trial. BMJ. Apr 1 2006;332(7544): 756-761.

131. Chenoweth L, King MT, Jeon YH, et al. Caring for Aged Dementia Care Resident Study (CADRES) of person-centred care, dementiacare mapping, and usual care in dementia: a cluster-randomised trial. Lancet Neurol. Apr 2009;8(4):317-325.

132. Edvardsson D, Winblad B, Sandman PO. Person-centred care of people with severe Alzheimer's disease: current status and ways forward. Lancet Neurol. Apr 2008;7(4):362-367.

133. Deudon A, Maubourguet N, Gervais X, et al. Non-pharmacological management of behavioural symptoms in nursing homes. Int J Geriatr Psychiatry. Dec 2009;24(12):1386-1395.

134. Teri L, Logsdon RG, Uomoto J, McCurry SM. Behavioral treatment of depression in dementia patients: a controlled clinical trial. J Gerontol B Psychol Sci Soc Sci. Jul 1997;52(4):P159-P166.

135. Cohen-Mansfield J. Nonpharmacologic interventions for inappropriate behaviors in dementia: a review, summary, and critique. Am J Geriatr Psychiatry. Fall 2001;9(4):361-381.

136. Turner S. Behavioural symptoms of dementia in residential settings: a selective review of non-pharmacological interventions. Aging Ment Health. Mar 2005;9(2):93-104.

137. McAllister CL, Silverman MA. Community formation and community roles among persons with Alzheimer's disease: a comparative study of experiences in a residential Alzheimer's facility and a traditional nursing home. Qual Health Res. Jan 1999;9(1):65-85.

138. Sloane PD, Mitchell CM, Preisser JS, Phillips C, Commander C, Burker E. Environmental correlates of resident agitation in Alzheimer's disease special care units. J Am Geriatr Soc. Jul 1998; 46(7): 862-869.

139. Zeisel J, Silverstein NM, Hyde J, Levkoff S, Lawton MP, Holmes W. Environmental correlates to behavioral health outcomes in Alzheimer's special care units. Gerontologist. Oct 2003;43(5):697-711.

140. Raglio A, Bellelli G, Traficante D, et al. Efficacy of music therapy in the treatment of behavioral and psychiatric symptoms of dementia. Alzheimer Dis Assoc Disord. Apr-Jun 2008;22(2):158-162.

141. Holmes C, Knights A, Dean C, Hodkinson S, Hopkins V. Keep music live: music and the alleviation of apathy in dementia subjects. Int Psychogeriatr. Dec 2006;18(4):623-630.

142. Guetin S, Portet F, Picot MC, et al. Effect of music therapy on anxiety and depression in patients with Alzheimer's type dementia: randomised, controlled study. Dement Geriatr Cogn Disord. 2009;28(1): $36-46$.

143. Svansdottir HB, Snaedal J. Music therapy in moderate and severe dementia of Alzheimer's type: a case-control study. Int Psychogeriatr. Dec 2006;18(4):613-621.

144. Gerdner LA. Top cited papers in International Psychogeriatrics: 4. Effects of individualized vs classical "relaxation" music on the frequency of agitation in elderly persons with Alzheimer's disease and related disorders. Int Psychogeriatr. Aug 2009;21(4):667-671.

145. Small BJ, MacDonald SWS, Iser L, Backman L. Memory and cognitive performance in preclinical Alzheimer's Disease and preclinical Vascular Disease. In: E Dere AE, Nadel L, Huston JP, editors. Handbook of Episodic Memory. New York: Elsevier; 2008: $537-552$.
146. Winblad B, Kilander L, Eriksson S, et al. Donepezil in patients with severe Alzheimer's disease: double-blind, parallel-group, placebocontrolled study. Lancet. Apr 1 2006;367(9516):1057-1065.

147. Backman L, Jones S, Berger AK, Laukka EJ, Small BJ. Cognitive impairment in preclinical Alzheimer's disease: a meta-analysis. Neuropsychology. Jul 2005;19(4):520-531.

148. Backman L, Jones S, Small BJ, Aguero-Torres H, Fratiglioni L. Rate of cognitive decline in preclinical Alzheimer's disease: the role of comorbidity. J Gerontol B Psychol Sci Soc Sci. Jul 2003;58(4): 228-236.

149. Palmer K, Backman L, Winblad B, Fratiglioni L. Detection of Alzheimer's disease and dementia in the preclinical phase: population based cohort study. BMJ. Feb 1 2003;326(7383):245.

150. Hultsch DF, Hertzog C, Dixon RA, Small BJ. Memory change in the aged. New York: Cambridge University Press; 1998.

151. Backman L, Forsell Y. Episodic memory functioning in a community-based sample of old adults with major depression: utilization of cognitive support. J Abnorm Psychol. May 1994;103(2): 361-370.

152. Calvaresi E, Bryan J. B vitamins, cognition, and aging: a review. J Gerontol B Psychol Sci Soc Sci. Nov 2001;56(6):P327-P339.

153. Wilson RS, Beckett LA, Barnes LL, et al. Individual differences in rates of change in cognitive abilities of older persons. Psychol Aging. Jun 2002;17(2):179-193.

154. Fahlander K, Wahlin A, Almkvist O, Backman L. Cognitive functioning in Alzheimer's disease and vascular dementia: further evidence for similar patterns of deficits. J Clin Exp Neuropsychol. Sep 2002;24(6): 734-744.

155. Thorvaldsson V, Macdonald SW, Fratiglioni L, et al. Onset and rate of cognitive change before dementia diagnosis: findings from two Swedish population-based longitudinal studies. J Int Neuropsychol Soc. Jan 2011;17(1):154-162.

156. Hall CB, Lipton RB, Sliwinski M, Stewart WF. A change point model for estimating the onset of cognitive decline in preclinical Alzheimer's disease. Stat Med. Jun 15-30 2000;19(11-12):1555-1566.

157. Hall CB, Derby C, LeValley A, Katz MJ, Verghese J, Lipton RB. Education delays accelerated decline on a memory test in persons who develop dementia. Neurology. Oct 23 2007;69(17): 1657-1664.

158. Smith GE, Pankratz VS, Negash S, et al. A plateau in pre-Alzheimer memory decline: evidence for compensatory mechanisms? Neurology. Jul 10 2007;69(2):133-139.

159. Stern Y. Cognitive Reserve: Theory and Applications. New York, NY: Taylor \& Francis; 2007.

160. Small BJ, Herlitz A, Fratiglioni L, Almkvist O, Backman L. Cognitive predictors of incident Alzheimer's disease: a prospective longitudinal study. Neuropsychology. Jul 1997;11(3):413-420.

161. Chen P, Ratcliff G, Belle SH, Cauley JA, DeKosky ST, Ganguli M. Patterns of cognitive decline in presymptomatic Alzheimer disease: a prospective community study. Arch Gen Psychiatry. Sep 2001;58(9): $853-858$.

162. Jagust W, Gitcho A, Sun F, Kuczynski B, Mungas D, Haan M. Brain imaging evidence of preclinical Alzheimer's disease in normal aging. Ann Neurol. Apr 2006;59(4):673-681.

163. Spulber G, Niskanen E, MacDonald S, et al. Whole brain atrophy rate predicts progression from MCI to Alzheimer's disease. Neurobiol Aging. Sep 2010;31(9):1601-1605.

164. Rowe CC, Ackerman U, Browne W, et al. Imaging of amyloid beta in Alzheimer's disease with 18F-BAY94-9172, a novel PET tracer: proof of mechanism. Lancet Neurol. Feb 2008;7(2):129-135.

165. Huang J, Auchus AP. Diffusion tensor imaging of normal appearing white matter and its correlation with cognitive functioning in mild cognitive impairment and Alzheimer's disease. Ann N Y Acad Sci. Feb 2007;1097:259-264.

166. Small BJ, Rosnick CB, Fratiglioni L, Backman L. Apolipoprotein $\mathrm{E}$ and cognitive performance: a meta-analysis. Psychol Aging. Dec 2004;19(4):592-600. 
167. Fratiglioni L, Paillard-Borg S, Winblad B. An active and socially integrated lifestyle in late life might protect against dementia. Lancet Neurol. Jun 2004;3(6):343-353.

168. Crowe M, Andel R, Pedersen NL, Johansson B, Gatz M. Does participation in leisure activities lead to reduced risk of Alzheimer's disease? A prospective study of Swedish twins. J Gerontol B Psychol Sci Soc Sci. Sep 2003;58(5):P249-P255.

169. Rovio S, Kareholt I, Helkala EL, et al. Leisure-time physical activity at midlife and the risk of dementia and Alzheimer's disease. Lancet Neurol. Nov 2005;4(11):705-711.
170. Grober E, Hall CB, Lipton RB, Zonderman AB, Resnick SM, Kawas C. Memory impairment, executive dysfunction, and intellectual decline in preclinical Alzheimer's disease. J Int Neuropsychol Soc. Mar 2008;14(2):266-278.

171. Jorm A. Prospects for the prevention of dementia. Australian Journal of Ageing. 2002;21:9-13.

172. Canada Alzheimer Society. Rising Tide: The Impact of Dementia on Canadian Society. 2010; www.alzheimer.ca/english/rising_tide/ rising_tide_summary.htm. Accessed January 23, 2011.

\section{Publish your work in this journal}

The Journal of Multidisciplinary Healthcare is an international, peerreviewed open-access journal that aims to represent and publish research in healthcare areas delivered by practitioners of different disciplines. This includes studies and reviews conducted by multidisciplinary teams as well as research which evaluates the results or conduct of such teams or healthcare processes in general. The journal covers a wide range of areas and welcomes submission from practitioners at all levels, from all over the world. The manuscript management system is completely online and includes a very quick and fair peer-review system. Visit http://www.dovepress.com/testimonials.php to read real quotes from published authors.

Submit your manuscript here: http://www.dovepress.com/journal-of-multidisciplinary-healthcare-journal 